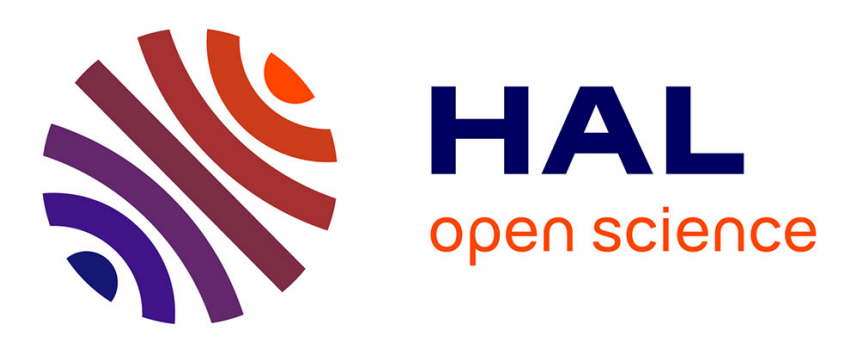

\title{
Analysis of the transitions between flow patterns in open-channel lateral cavities with increasing aspect ratio
}

Emmanuel Mignot, Wei Cai, Nicolas Rivière

\section{To cite this version:}

Emmanuel Mignot, Wei Cai, Nicolas Rivière. Analysis of the transitions between flow patterns in open-channel lateral cavities with increasing aspect ratio. Environmental Fluid Mechanics, 2019, 19 (1), pp.231-253. 10.1007/s10652-018-9620-x . hal-02381227

\section{HAL Id: hal-02381227 \\ https://hal.science/hal-02381227}

Submitted on 29 Nov 2019

HAL is a multi-disciplinary open access archive for the deposit and dissemination of scientific research documents, whether they are published or not. The documents may come from teaching and research institutions in France or abroad, or from public or private research centers.
L'archive ouverte pluridisciplinaire HAL, est destinée au dépôt et à la diffusion de documents scientifiques de niveau recherche, publiés ou non, émanant des établissements d'enseignement et de recherche français ou étrangers, des laboratoires publics ou privés. 


\title{
Analysis of the transitions between flow patterns in open-channel lateral cavities with increasing aspect ratio
}

\author{
Emmanuel Mignot $^{1 *}$, Wei Cai ${ }^{1}$, Nicolas Riviere $^{1}$ \\ Informative title: Flow pattern in lateral cavities \\ ${ }^{1}$ Univ Lyon, INSA Lyon, CNRS, LMFA UMR5509, F-69621 Villeurbanne, France. \\ * corresponding author: Emmanuel.mignot@insa-lyon.fr,0033-472438070.
}

ABSTRACT: The flow pattern within a storage zone governs the efficiency of passive scalar accumulation, spreading and homogenization. The present paper aims at characterizing experimentally the evolution of this flow pattern in a simplified open-channel lateral cavity with twenty increasing horizontal aspect ratio, focusing on the transition between the different flow patterns. Four main flow types are observed and detailed herein with, as the aspect ratio gradually increases: two cells aligned along the main stream axis, one unique cell, two cells aligned transversally and finally a more complex 3D pattern in the second cell. Quantitative characteristics of these cells are extracted from the velocity fields measured in the cavity. A simplified 2D torque balance is then applied to explain the transitions between these flow types and proves to be able to predict the flow patterns reported in the literature. Finally, we show that the flows in the cavity exhibit low frequency motions of about $1 \mathrm{Hertz}$, related to turbulent structures shed at the interface with the main stream and entering the cavity.

Keywords: Cavity, Flow pattern, Recirculation cells, Analytical model, Experiments

\section{Introduction}

Lateral cavities in riverine environments are major storage zones for pollutions, nutrients, dissolved gazes or sediments transported by the river stream from upstream areas (Ensign and Doyle, 2005; Argerich et al., 2011). This material is transferred towards the cavity through the mixing layer that forms at the cavity/main stream interface, by turbulent diffusion (Mignot et al., 2016). It then spreads within the cavity through advection by the recirculation cells and through turbulent diffusion from one cell to another and within the cells themselves (Mignot et al., 2017). These authors showed that the flow pattern within the cavity and particularly the number, size and typical velocity magnitude of each cell governs the mass transfer from the main stream to the storage zone and within the storage zone. The present paper is then devoted to gain information on these flow patterns that develop in lateral cavities adjacent to rivers, focusing on the transitions between the flow types.

Such investigation in laboratory conditions is usually performed by highly simplifying the geometry of both the main stream and the cavity to obtain generic conditions. Most studies (including those in 
Table 1) consider main streams on horizontal and smooth beds with rectangular cross-sections connected to a rectangular cavity limited by vertical smooth walls with $90^{\circ}$ sharp angles (as in Fig.1). Still, a few studies in the literature consider more field-like geometries such as a wall that partially blocks the interface between the cavity and the main stream ( $\mathrm{Li}$ and Ip, 1999; Nezu and Onitsuka, 2002b), non-rectangular shape cavities (Jackson et al., 2015; Tominaga \& Jong, 2011), a vertical step at the interface (Muto et al., 2000) or a longitudinal slope covering the bottom of the cavity (Sanjou and Nezu, 2013). In these more complex configurations, the flow pattern is strongly dependent on the geometrical details so that these configurations will not be used for comparison with the present results and are excluded from Table 1.

Two main configurations of simplified side cavity can be found in the literature: the so-called "lateral cavity" (as Fig.1) and "cavity between two groynes". The main difference between these two configurations lies in the direction of the flow when reaching the entrance of the cavity: for the lateral cavity, the flow reaching the upstream corner is parallel to the channel axis and so also to the main stream/cavity interface. Oppositely, in a cavity located between two groynes, the approaching flow is slightly oriented away from the cavity, towards the center of the main stream. In Table 1, the two configurations are listed separately even though the corresponding flow patterns appear to be in fair agreement. Note that in the case of fields of consecutive groynes or cavities, an additional difference is the level of development of the mixing layer when reaching the dead-zone: in a groyne/cavity field the mixing layer already started to develop in the cavities located upstream while for a single groyne/cavity, the mixing layer only depends on the development of the boundary layer along the upstream lateral wall of the main stream. Table 1 reveals that the mean flow pattern is primarily dependent on the geometrical aspect ratio of the cavity: $W / L$ with $W$ its width and $L$ its length (see Fig.1), even though inconsistencies exist between the available flow pattern in terms of number of recirculating cells with regards to aspect ratio of the cavity, probably due to the impact of the other dimensionless parameters (see Mignot et al., 2017):

- For cavities with a small aspect ratio $(0.2<W / L<0.33)$, a strongly distorted single cell of vertical axis occupies most of the available volume of the cavity.

- For cavities with $0.3<W / L<0.6$, two contra-rotating recirculation cells are aligned longitudinally, i.e. along the channel axis (along $x$ axis) but with the most upstream one being of smaller horizontal extension and located further from the interface (higher $y$ value).

- For cavities with larger geometrical aspect ratio $(0.5<W / L<1.5)$ a single cell, about circular, occupies the available volume of the cavity.

- For longer cavities with $2<W / L<3$, two contra-rotating recirculation cells are aligned perpendicularly to the interface (along $y$ axis). 
Numerous studies were dedicated to other types of cavities that exhibit similarities to the present openchannel lateral cavities. Open cavities connected to air flows are of major interest in aerodynamics, the reader may refer to the recent publication by Douay et al. (2016) for more details. Lid-driven cavities are highly simplified configurations, of much use for the validation of numerical models; for more information, the reader may refer to the publication by Peng et al. (2003). Among these works, some dealt specifically with the flow patterns and corresponding transitions as a function of the cavity width to length ratio. Faure et al. (2007) visualized the flow, using smoke and a laser sheet, in an open cavity located below an air-flow, i.e. without a free-surface. The authors report, as the aspect ratio of the cavity increases (in the direction perpendicular to the main flow axis): i) two contra-rotating cells aligned with the main flow axis, ii) a single cell occupying the whole cavity for a square cavity and iii) two cells aligned in the direction perpendicular to the main flow axis. Regarding lid-driven cavities, authors such as Taneda (1979), Shankar and Deshpande (2000) or Lin et al. (2011) measured or computed the flow patterns for various Reynolds numbers and aspect ratios of the cavity. They also report that increasing the aspect ratio of the cavity (in the direction perpendicular to the moving boundary) permits to pass from i) two cells aligned along the rigid lid direction, to ii) a single cell and to iii) several cells aligned perpendicularly. Flow patterns with up to 4 aligned contra-rotating cells are then reported for very long cavities. These flow patterns are then in fair agreement with the flow patterns listed above for an open-channel lateral cavity. However, present flow patterns strongly differ due to the presence of a free-surface.

The studies listed in Table 1 give a fair overview of the flow patterns forming in open-channel lateral cavities, but only for sparse and discrete values of $W / L$, and all of them being smaller than 3 . Thus, the present study aims at (i) extending this previous knowledge to aspect ratios higher than 3 and up to 5, (ii) characterizing the transition between the different flow patterns through velocity measurements and the application of an analytical torque model and (iii) characterizing the time evolution of these flow patterns, related to the turbulent activity in the cavity. As the paper focuses on the transitions between the different flow patterns, the strategy employed is to keep a constant mean flow (water depth and mean velocity in the main stream) while gradually increasing the geometrical aspect ratio of the cavity by increasing its width $W$. The next section first describes the experimental facility and the measurement devices used herein and introduces the parameters that permit to quantify the characteristics of the recirculation cells. The analytical torque model is then introduced and applied to the present and literature data in the following section. The last section is devoted to the steadiness/time evolution of these flow patterns, associated with turbulent structures shed and advected within the cavity.

\section{Experimental approach}




\subsection{Experimental set-up and measurement methods}

The experiments detailed herein are performed at the Laboratoire de Mecanique des Fluides et d'Acoustique at the Université de Lyon, France. The cavity facility consists of a $4.9 \mathrm{~m}$ long and $b=0.3 \mathrm{~m}$ wide horizontal open-channel, of rectangular cross section, made of glass and connected at mid length with a rectangular cavity. The length of the cavity in the direction parallel to the main stream is fixed and equals $L=b=0.3 \mathrm{~m}$, while its width $W$ in the direction perpendicular to the main stream can be varied continuously up to $W / L=5$, as depicted in Fig.1. The inlet discharge in the main stream is fixed to $Q=0.0035 \mathrm{~m}^{3} / \mathrm{s}$, measured using a Promag 50 flowmeter (from Endress Hauser; with an uncertainty of $5 \times 10^{-5} \mathrm{~m}^{3} / \mathrm{s}$ ) within the pumping loop. A honeycomb mesh is installed at the inlet of the channel in order to stabilize and straighten the inflow, and a sharp crested weir at the outlet is used to fix the water depth to $h=0.07 \mathrm{~m}( \pm 0.15 \mathrm{~mm})$ at the entrance of the cavity. It is verified using a point gauge that the maximum under-elevation and free-surface oscillations in the cavity always remain negligibly small, with an amplitude smaller than $1 \%$ of the water depth. The mean velocity in the main stream thus equals $U=Q /(b h)=0.166 \mathrm{~m} / \mathrm{s}$, so that the Reynolds number of the flow is $\mathrm{Re}=$ $U D_{h} / v=4 Q /[v(b+2 h)]=3.2 \times 10^{4}$ and the Froude number is $\mathrm{Fr}=U /(g h)^{0.5}=0.2$, with $D_{h}$ the hydraulic diameter of the flow in the channel and $v$ the kinematic viscosity of water. On the other hand, the Reynolds number associated to the cavity flow is about $\operatorname{Re}_{c}=U_{c} L / v \sim 8000$ with $U_{c}$ the typical velocity magnitude along the cavity-main stream interface.

The horizontal velocity fields are measured using a 2D horizontal PIV method, permitting to access the $u$ and $v$ mean components of the velocity along the Cartesian $x$ and $y$ axes respectively at a given elevation, as shown in Fig.1. Most velocity fields presented herein are measured at the elevation $z_{L}=0.05 \mathrm{~m}\left(z_{L} / h=0.71\right)$, that is sufficiently far from the bed to expect any dependency to the measurement elevation (as discussed by Tuna et al., 2013). Additional measurement elevation planes are considered in a specific figure in the sequel. Polyamid particles with a diameter of $50 \mu \mathrm{m}$ (density $1030 \mathrm{~kg} / \mathrm{m}^{3}$ ) are added to the water within the recirculation loop, and a $40 \mathrm{~mW}$ continuous laser coupled with a cylindrical lens generates a $1 \mathrm{~mm}$ thick, horizontal light sheet at the desired elevation. A video camera located above the cavity (at an elevation of 1.5 to $1.7 \mathrm{~m}$ above the free-surface) records the motion of the particles for over 3000 consecutive images, using a resolution of $1280 \times 960$ pixels with an average spatial resolution of $0.8 \mathrm{~mm}$ per pixel. The image recording frequency along with the location of the camera and number of measured domains depend on the geometry of the cavity:

- for narrow cavities $(W / L \leq 2.3)$, the laser is located on the opposite side of the channel (location A in Fig.1a), and the camera is centered above the center of the cavity. A single video records the particle motions over the whole measurement domain with a single-frame sampling frequency equal to $12.2 \mathrm{fps}$ (frames per second), leading to a measurement duration of $3000 / 12.2=246$ seconds. 
- for wider cavities ( $W / L \geq 2.6)$, a single video does not permit to measure the velocity field over the whole domain with a high enough spatial resolution. Two videos are then captured. The first video is obtained in the same condition as for the narrow cavities (laser location A, single-frame sampling frequency $12.2 \mathrm{fps}$, duration 246 seconds, covering the region $0<y / L<3$, see Fig.1b). The second video covers the remaining area of the cavity $(2<y / L<W / L)$ with the camera located above this area and the laser located on the opposite side of the cavity (location B in Fig.1b). The single-frame sampling frequency is adapted to the lower velocity of the second recirculation cell and is lowered to $3.75 \mathrm{fps}$, leading to a measurement duration of $3000 / 3.75=800$ seconds.

In all cases, the commercial software Davis (from LaVision) is used to correct image distortions, subtract the background from the images and compute each instantaneous velocity field with a grid resolution of $5 \mathrm{~mm}$, i.e. 60 measurement points per cavity length $L$. Note that the post-processing only considers the velocity vectors for which the main correlation peak within the interrogation window exceeds 1.2 times the second largest correlation peak; an average of $0.7 \%$ of the number of velocity vectors is then considered false vectors, i.e. non-measured velocity data. The flow field is then timelyaveraged after checking that the time convergence is verified for each cell, as discussed in the last section. For the wider cavities with two videos, the time-averaged velocity fields are combined and the flow field in the overlap region $(2<y / L<3)$ is obtained using a weighted averaging process.

In order to estimate the uncertainty of the measured velocity fields, it is first verified that the Stokes number associated to the seeding particles remains low enough $(\mathrm{St} \approx 0.05)$ to assume that the particles follow the flow closely enough. Then, as for Jahanmiri (2011) and Mignot et al. (2014 and 2016), the Gaussian peak fitting process is assumed to generate a flow displacement uncertainty equal to 0.1 pixels which should be added to other sources of uncertainty, mainly due to any seeding concentration gradient in the fluid. This gradient being insignificant herein, we assume, as for Jahanmiri (2011), that this second source of uncertainty leads to an uncertainty of about the same magnitude as for the peak fitting process. Consequently, the uncertainty associated to the velocity measurement equals about 2 $\mathrm{mm} / \mathrm{s}[2 \times 0.1$ pix $\times$ spatial resolution $(0.8 \mathrm{~mm} /$ pix $) \times$ acquisition frequency $(12.2 \mathrm{~Hz})]$ for the measurements in the narrow cavities or for $y / L<2.5$ in the wide cavities and equals about $0.6 \mathrm{~mm} / \mathrm{s}$ [ $2 \mathrm{x}$ 0.1 pix x $0.8 \mathrm{~mm} /$ pix x $3.75 \mathrm{~Hz}$ ] for $y / L>2.5$ in the wide cavities. Moreover, the fair agreement between the measured velocities in the overlap region from both videos in the case of wide cavities and the high degree of reproducibility of the measurements give additional confidence to the measured velocities.

The incoming velocity field is additionally measured in the inlet branch of the main stream $(x<0$ and $y<0$ ). Fig. 2 shows transverse profiles (along $y$ axis) of streamwise (along $x$ axis) time-averaged velocity in the lateral boundary layer attached to the right lateral wall at two sections approaching the 
cavity region. This figure reveals that the incoming flow field is quite established before reaching the cavity region.

\subsection{Definition of the geometrical parameters}

As discussed above (see Table 1), the mean flow patterns in the lateral cavities consist in one or two 2D horizontal recirculation cells. In order to define the location and length of each recirculation cell and the associated recirculation velocity, the following parameters are defined.

First, the center of each recirculation cell (points 1 and 2 in Fig. 3) is defined as the location of minimum local velocity magnitude. $X \mathrm{c}_{1}$ and $X \mathrm{c}_{2}$ are then defined as the distances (along $x$ ) between these centers and the downstream wall of the cavity. $Y \mathrm{c}_{1}$ and $Y \mathrm{c}_{2}$ are the distances (along $y$ ) from the centers to the interface with the main stream for $Y \mathrm{c}_{1}$ and to the opposite wall for $Y \mathrm{c}_{2}$.

Second, the frontier between cells 1 and 2 (along the downstream and upstream wall) is characterized by points 3 and $4 . Y_{3}$ is defined as the distance along the downstream wall from the interface to the location where the velocity component $v$ (along $y$ axis) changes sign from positive (in the first cell) to negative (in the second cell), see Fig. 3b. Then, if the second cell is confined near the downstream/opposite wall corner (as in Fig.3a), $X_{4}$ is defined as the distance along the opposite wall from the downstream wall to the location where the velocity component $u$ (along $x$ axis) changes from positive (in the second cell) to negative (in the first cell), see Fig.3a. Oppositely, if the second cell occupies the whole length of the cavity (from $x / L=0$ to 1 as in Fig. $3 b$ ), $Y_{4}$ is defined as the distance along the upstream wall from the interface to the location where $v$ changes sign from negative (in the first cell) to positive (in the second cell), see Fig.3b.

\section{Velocity fields in cavities with increasing geometric aspect ratios}

Data in Table 1 reveal that the number and alignment of recirculation cells pattern in a lateral cavity are highly dependent on its geometrical aspect ratio. To gain information on these cells, Fig.4 plots the velocity fields, measured at the elevation $z / L=0.71$, for twenty cavities ranging from a narrow cavity oriented along the main stream axis $(W / L=0.3)$ to a wide cavity oriented perpendicularly $(W / L=5)$, Fig.5 shows the evolution of the geometrical parameter values and Fig.6 exhibits the mechanisms associated to the transition from a one-cell to a two-cell configuration.

Fig.4 confirms that for a narrow cavity $(W / L=0.3)$ two horizontal contra-rotating cells are aligned along $x$ axis, for slightly wider cavities $(0.6<W / L<2)$ a single cell occupies most of the available area with two small additional secondary cells confined in the opposite corners $(y / W=1)$, for still wider cavities $(2.2<W / L<3)$ two adjacent contra-rotating cells are aligned along $y$ axis (also with two small additional secondary cells confined in the opposite corners, at $y / W=1$ ), and finally, for the widest cavities $(W / L>3)$ this second cell becomes slightly 3D. These patterns are in fair agreement with the open-channel side cavity flows referred to in Table 1 but are also in qualitative agreement with the lid- 
driven-cavities such as those presented by Cheng and Hung (2006) in their figure 8 (with a Reynolds number $\operatorname{Re}_{\mathrm{c}}=1000$ of same order of magnitude as the present one) as long as a maximum of two vortices are observed in the $y$ direction.

The maximum velocity magnitude is measured along the longest lateral walls (or interface), i.e. along the opposite wall and the interface for $W / L<1$ and along the upstream and downstream walls for the wider cavities. Moreover, for cavities with $W / L>0.6$, the typical velocity of the first cell does not evolve much as their geometrical aspect ratio increases. A remarkable result is the high similarity of velocity magnitude distribution with $W / L=1.6$ and in the first cell for $W / L=5$ in Fig.4. On the other hand, the typical velocity of the second cell equals about $0.5 \mathrm{~cm} / \mathrm{s}$ for $2.3<W / L<3$ (that is about a third of that of the first cell, i.e. $0.03 \mathrm{U}$ ) and lowers to about $0.1 \mathrm{~cm} / \mathrm{s}$ (about $1 / 15$ of the first cell velocity, i.e. $0.006 \mathrm{U}$ ) for the widest cavities with $W / L>=3.6$.

Fig.5 reveals that as long as only one cell is measured $(0.6<W / L<2)$, its center point is located at about mid-length of the cavity along $x$ and $y$ axes, but slightly closer to the downstream wall so that $X \mathrm{c}_{1} / L \sim 0.45$ while $Y_{\mathrm{C} 1} / W \sim 0.5$. Oppositely, for the two-cells wide cavities ( $W / L>2.3$ ), the location of the center and the length of the first cell vary little for increasing aspect ratios of the cavity: $Y_{3} / L \sim Y_{4} / L \in[1.5-1.9]$ and $Y_{\mathrm{c}_{1}} / L \in[0.8-0.9]$. In such case, the second cell occupies the remaining available space so that $Y \mathrm{c}_{2} / L$ increases about linearly with $W / L$ as long as the flow remains $2 \mathrm{D}$ $(2.3<W / L<3)$. Oppositely, for the more 3D flow configurations in the widest cavities $(W / L>3)$, the distance from the interface to point 2 does not change anymore, which explains the increasing rate of 1 for the curve $Y \mathrm{c}_{2} / L=\mathrm{f}(W / L)$. Besides, $X \mathrm{c}_{2}$ remains about constant $\left(X \mathrm{c}_{2} / L \sim 0.5\right)$ for the $2 \mathrm{D}$ flow configurations with two cells $(2.3<W / L<3)$ but decreases for the widest cavity with $3 \mathrm{D}$ flows $(W / L>3)$ with the center of the second cell approaching the downstream wall.

The transition from a cavity with one cell to two transversally-aligned cells appears to occur for the present configuration for $W / L=2$ to 2.3 (see Fig.4). Fig.6 then plots (for three configurations in this range: $W / L=2,2.2$ and 2.3) consecutive mean flow patterns every 10 seconds, each one averaged over 10 seconds (122 samples). For the narrower configuration $W / L=2$, the second cell appears to remain confined near the downstream/opposite wall corner at all time as for its time-averaged flow pattern and for the flow patterns with lower aspect ratios in Fig.4. Oppositely, for the wider configuration $W / L=2.3$, the second cell occupies the whole cavity length (from $x / L=0$ to 1 ) at all time, as for the corresponding time-averaged flow and the flow patterns with higher aspect ratios in Fig.4. For the intermediate configuration $W / L=2.2$, the flow alternates along the time between these two flow patterns without clear tendency: the second cell is sometimes confined in the corner (as for $t=90-100 \mathrm{~s}$ or $t=10-30 \mathrm{~s}$ ) and sometimes occupies the whole cavity length (as for $t=140-170 \mathrm{~s}$ ).

\section{Analytical torque model of the flow in the cavity}




\subsection{Model description}

The present section aims at identifying the origin of the transition detailed in the previous section from two cells aligned longitudinally for a narrow cavity, to a single cell for a cavity with an averaged aspect ratio, and finally to two cells aligned transversally for the widest cavities. The approach detailed below is an extension of Hill (2014)'s work who applied an equilibrium torque balance in order to estimate the mean recirculation velocity in a square cavity $(W / L=1)$. This balance reads:

$T_{i}=T_{w}+T_{b}+T_{p}$

where $T_{i}$ is the forcing torque that the main stream applies on the cavity flow at the interface with regards to the center of the cavity, $T_{w}$ and $T_{b}$ are the resisting torques due to friction respectively on the three lateral walls and on the bottom of the cavity and $T_{p}$ is the resulting torque due to pressure forces (neglected by Hill, 2014). As Hill (2014) semi-empirical closures are only valid for square cavities, the present strategy extends this method to narrower and wider rectangular cavities.

Let the four velocity parameters $U_{i}, U_{d}, U_{o}$ and $U_{u}$ be the velocity magnitudes near respectively the center of the interface, the downstream, the opposite and the upstream walls, see Fig.7. Fig.8 plots velocity profiles along both horizontal axes across the cavity, for increasing aspect ratios $W / L$. It appears that within the first cell the velocity magnitude increases about linearly in the four perpendicular directions: from the center of the cell towards each wall (or the interface). These velocity profiles are in agreement with the rigid-lid cavity flows measured by Koseff and Street (1984) in their figure $3 \mathrm{~b}$ with a Reynolds number of $\mathrm{Re}_{\mathrm{c}}=10^{4}$ and computed by Cheng and Hung (2006) in their figure 5 with $\mathrm{Re}_{\mathrm{c}}=5000$ (both about similar to our $\operatorname{Re}_{\mathrm{c}} \sim 8000$ configuration). This shows that the classical rigid body rotation assumption fairly reproduces the water motion in the first cell of the cavity, at least up to the vicinity of the walls. Moreover, a striking result on Fig.8b is that for $W / L \geq 1$, the velocity profiles along the short axis $(x)$ are all similar, indicating that $U_{u} \sim U_{d} \sim K U$ with $K=0.2$ herein for all $W / L$ values. Note that the reason for the velocity magnitudes near the downstream wall $\left(U_{d}\right)$ to slightly exceed that near the upstream wall $\left(U_{u}\right)$ is due to the fact that the center of the cell is slightly shifted towards the downstream wall: $X_{\mathrm{c} 1} / L<0.5$, as observed in Fig.5. The consequences of i) the rigid body rotation and ii) the similar velocity profiles from the center of the cell towards the upstream and downstream walls are twofold: (1) the entrained discharge rotating in the first cell $Q_{E}$ remains constant for $1<W / L \leq 2$ with $Q_{E}=K U h L / 4=0.05 K U h L$ and (2) $U_{o}$ and $U_{i}$ are also about equal to each other for a given $W / L$ ratio with $U_{o} \sim U_{i} \sim U_{d} L / W \sim K U L / W$ (Fig.8c).

Additionally, in order to verify that the flow in the $1^{\text {st }}$ cell can be considered as $2 \mathrm{D}$ in this model, Fig.9 shows horizontal velocity fields measured at different elevations for a selected configuration: $W / L=3$. It appears that the general flow pattern does not vary along the vertical axis, with two cells of same dimensions and locations form at all measured elevations. The characteristics of the first cell do not change much along the water column, in agreement with observations by Tuna et al. (2013) while 
those of the second cell are more affected: in the upper part of the water column, the streamline separating both cells follows an oblique direction, oriented from low to higher $y$ values for decreasing $x$ values while in the lower part of the water column, it is oriented from high to lower $y$ values. In the near bed region $(z / h=0.21$ and below), a saddle point appears at the interface between both cells $(y / L=1.7$ for $W / L=3$ and $y / L=1.9$ for $W / L=5)$, close to the upstream wall $(x / L \sim 0.2)$ accompanied by a "half node of separation" at the adjacent wall $(x=0)$ using the definition proposed by Younis et al. (2014). Oppositely, further up in the water column, this node is replaced by a more simple "half saddle of attachment" at the wall (also referred to as "reattachment point"). These results thus justify the use of a 2D horizontal torque model.

Finally, the main result that will be used in the following model is that all velocity profiles in the first cell can be approximately deduced from the extension of the first cell and the mean flow bulk velocity. In other words, for $1<W / L<2$ :

$\left\{U_{u} / U \sim U_{d} / U \sim \mathrm{K}\right.$

$\left\{U_{o} / U \sim U_{i} / U \sim K L / W\right.$

with $K=0.2$ herein. Using these results, the four terms of Eq. 1 can be evaluated in the following subsections.

\section{Interface forcing torque $T_{i}$}

Using a mixing length approach, the horizontal Reynolds shear stress along the interface mixing layer reads:

$\left|\tau_{x y}\right|=\rho L_{m}^{2}\left|\frac{\partial U}{\partial y}\right|^{2}$

with $\rho$ the water density, $L_{m}=0.07 \delta$ (Rodi, 1993) the mixing length, $\delta$ the mixing layer width (see Mignot et al., 2016 and 2017) and $|\partial U / \partial y| \sim\left(U-U_{i}\right) / \delta \sim U(1-K L / W) / \delta$, using Eq.2. Consequently, assuming a symmetric elliptic rigid body rotation in the cavity, the interface torque reads:

$T_{i}=\underbrace{\rho 0.07^{2} U^{2}\left(1-K \frac{L}{W}\right)^{2}}_{\left|\tau_{x y}\right|} \underbrace{h L}_{S_{i}} \underbrace{\frac{W}{2}}_{l_{i}}$

with $S_{i}$ the surface of the interface and $l_{i}$ the corresponding lever arm. The evolution of $T_{i}$ with increasing $W / L$ in the present configuration is plotted in Fig.10 and will be discussed further on. Note that, instead of using a constant velocity $U_{i}$ in Eq. 4 , an alternative method can be to use the actual measured velocity profile along the interface $u(x, y=0)$ to estimate the torque. The interface torque then reads (assuming a $2 \mathrm{D}$ flow):

$T_{i 2}=\rho 0.07^{2} h \frac{W}{2} \int_{x=0}^{x=L}(U-u(x, y=0))^{2} \mathrm{~d} x$ 
The resulting torque is also plotted in Fig.10. Both estimates appear to be in fair agreement with each other, giving confidence to the predictive formulation of Eq.4.

\section{Resisting torques}

Following Hill (2014) approach, let's assume that the flow in the cavity forms in a horizontal ellipse (rather than a rectangle) with axes of length $W / 2$ and $L / 2$. Let $R$ be the distance from the center (o) to a given point $p$ in the cavity (see Fig.7), and $0<e<1$ be the ellipse eccentricity (ratio between $R$ and the radius of the outer ellipse along the direction $o-p)$. Let $c(x, y)$ be the local time averaged velocity magnitude at point $p$. The rigid body rotation and Eq. 2 permit to relate $c$ to the main stream velocity $U$ as:

$c=U_{d} \frac{L \cdot e^{2}}{2 R}=K U \frac{L \cdot e^{2}}{2 R}$

Moreover, it can be geometrically shown that (see Fig.7)

$$
\left\{\begin{array}{c}
R=\frac{e W}{2 \sqrt{\left(\frac{W}{L}\right)^{2} \cos ^{2} \theta+\sin ^{2} \theta}} \\
\varphi=\theta-\operatorname{atan}\left[\left(\frac{L}{W}\right)^{2} \tan (\theta)\right]
\end{array}\right.
$$

with $\theta$ the angle between segments $o-r$ (parallel to $x$ axis) and $o-p$ and with $\varphi$ the angle between $o-q$ and $o-p, q-p$ being tangent to the ellipse at point $p$ while $o-q$ and $q-p$ being perpendicular to each other (see Fig.7). Using Eqs. 6-7, the local velocity $c$ reads

$$
c=K U e\left(\frac{L}{W}\right) \sqrt{\left(\frac{W}{L}\right)^{2} \cos ^{2} \theta+\sin ^{2} \theta}
$$

\section{Lateral wall resisting torque}

$T_{\mathrm{w}}$ is the resisting torque due to friction along the three lateral walls bounding the cavity, i.e. upstream, opposite and downstream walls, approximated by the outer ellipse discussed above (with $e=1$ ). The local wall shear stress reads $\tau_{\mathrm{w}}=\rho c^{2} C_{w} / 2$, with $C_{w}$ the wall friction coefficient. Using Eqs.7-8, the fact that $e=1$ and with $\mathrm{d} S$ the elementary surface and $l_{p}$ the lever arm, the wall resisting torque per unit wall surface $\mathrm{d} T_{w}$ reads:

$d T_{w}=\underbrace{\frac{1}{2} \rho c^{2} C_{w}}_{\tau_{w}} \underbrace{\operatorname{Rd} \theta \mathrm{dz}}_{d S} \underbrace{\operatorname{Rcos}(\varphi)}_{l_{p}}=\frac{C_{w} \rho(K U)^{2} L^{2} \cos (\varphi)}{8} \mathrm{~d} \theta \mathrm{dz}$

Integrating this elementary torque over the surface of the outer ellipse ( $z$ ranging from 0 to $h$ and $\theta$ ranging from $-\pi / 2+\psi$ to $3 \pi / 2-\psi$, see Fig.7, or for more simplicity two times $\theta$ ranging from $-\pi / 2+\psi$ to $\pi / 2$ ) leads to the wall resisting torque: 


$$
\begin{aligned}
T_{w} & =2 \int_{0}^{h} \int_{-\frac{\pi}{2}+\psi}^{\frac{\pi}{2}} \frac{C_{w} \rho(K U)^{2} L^{2} \cos \left[\theta-\operatorname{atan}\left(\frac{\tan (\theta)}{(W / L)^{2}}\right)\right]}{8} \mathrm{~d} \theta \mathrm{dz} \\
& =\frac{C_{w} \mathrm{~h} \rho(K U)^{2} L^{2}}{4} \int_{-\frac{\pi}{2}+\psi}^{\frac{\pi}{2}} \cos \left[\theta-\operatorname{atan}\left(\frac{\tan (\theta)}{(W / L)^{2}}\right)\right] \mathrm{d} \theta
\end{aligned}
$$

Eq.10 has unfortunately no analytical solution, as can be checked on its simplified form obtained by substituting $F=\tan ^{2}(\theta)$ :

$T_{w}=\left[\frac{C_{w} \mathrm{~h} \rho(K U)^{2} L^{2}}{4}\right] \frac{1}{2} \int_{F=\tan ^{2}\left(\psi-\frac{\pi}{2}\right)}^{F=\infty} \frac{(W / L)^{2}+F}{\sqrt{F\left[(W / L)^{4}+F\right](1+F)^{3}}} \mathrm{dF}$

Eq.10 is thus solved numerically. The evolution of $T_{w}$ with $W / L$ is plotted in Fig.10. Note that for a square cavity ( $W / L=1, \psi=\pi / 4)$, Eq.10 reduces to equation 5 from Hill (2014) with $C_{w}=C_{d w}\left(u_{g} / K U\right)^{2}$, where $u_{g}$ is the "velocity at the edge of the gyre" as defined by these authors.

\section{Bottom resisting torque}

The local bottom shear stress reads $\tau_{\mathrm{b}}=\rho c^{2} C_{b} / 2$ (in the opposite direction from the local velocity, i.e. along $p-q$ ) with $C_{b}$ the bed friction coefficient. Using Eqs.7-8, with $\mathrm{d} S$ the elementary surface and $l_{p}=|\overrightarrow{o q}|$ the lever arm, the local bottom resisting torque per unit surface $\mathrm{d} T_{b}$ reads:

$d T_{b}=\underbrace{\frac{1}{2} \rho c^{2} C_{b}}_{\tau_{b}} \underbrace{\operatorname{Rd} \theta \mathrm{dR}}_{d S} \underbrace{\operatorname{Rcos}(\varphi)}_{l_{p}}=\frac{\rho(K U)^{2} e^{4} L^{2} W C_{b} \cos (\varphi)}{16 \sqrt{\left(\frac{W}{L}\right)^{2} \cos ^{2} \theta+\sin ^{2} \theta}} \mathrm{d} \theta \cdot \mathrm{de}$

Integrating this elementary torque over the bottom surface of the ellipse (with $e$ ranging from 0 to 1 and $\theta$ from 0 to $2 \pi$, i.e. 4 times $\theta$ ranging from 0 to $\pi / 2$ ) leads to the bottom resisting torque:

$$
\begin{gathered}
T_{b}=\frac{\rho(K U)^{2} L^{2} W C_{b}}{16}\left\{\int_{0}^{1} e^{4} \operatorname{de}\right\}\left\{\int_{0}^{\pi / 2} 4 \frac{\cos \left[\theta-\operatorname{atan}\left(\frac{\tan (\theta)}{(W / L)^{2}}\right)\right]}{\sqrt{\left(\frac{W}{L}\right)^{2} \cos ^{2} \theta+\sin ^{2} \theta}} \mathrm{d} \theta\right\} \\
=\frac{\rho(K U)^{2} L^{2} W C_{b}}{20}\left\{\int_{0}^{\pi / 2} \frac{\cos \left[\theta-\operatorname{atan}\left(\frac{\tan (\theta)}{(W / L)^{2}}\right)\right]}{\sqrt{\left(\frac{W}{L}\right)^{2} \cos ^{2} \theta+\sin ^{2} \theta}} \mathrm{d} \theta\right\}
\end{gathered}
$$

Again, Eq.13 was solved numerically as no analytical solution could be obtained. Note that for a square cavity ( $W / L=1)$, Eq.13 reduces to equation 6 from Hill (2014) (who uses a moment coefficient $C_{m b}$ available for $W / L=1$ in the literature) with $C_{b}=5\left(u_{g} / K U\right)^{2} C_{m b} / 8$. The evolution of $T_{b}$ with $W / L$ is included in Fig.10.

\section{Torque due to pressure forces}

As the mean flow reaches the downstream edge of the interface $(x=L, y=0)$, an overpressure $\mathrm{d} P$ is generated there with $\mathrm{d} P \approx \rho U_{i}^{2} / 2=\rho(K U L / W)^{2} / 2$. This overpressure generates a forcing torque along the interface and a resisting torque along the downstream wall (see Fig. 7). Assuming a hydrostatic vertical pressure distribution and a linear decrease of this overpressure over a distance 
equal to $L / 2$ along both the interface and the downstream wall (as schematized by grey triangles filled with arrows on Fig. 7), the resulting (positively resisting) pressure torque reads:

$T_{p}=\frac{\rho h(W-L) K^{2} U^{2} L^{3}}{16 W^{2}}\left[1+\frac{K^{2} U^{2} L^{2}}{6 g h W^{2}}\right] \approx \frac{\rho h(W-L) K^{2} U^{2} L^{3}}{16 W^{2}}$

This term is finally included with all others in Fig. 10.

\subsection{Applications of the model to the present experimental configurations}

To apply this model, one main challenge lies in the choice of the wall and bottom friction coefficients $C_{w}$ and $C_{b}$. Regarding $C_{w}$, Hill (2014) proposed to assimilate the flow in the vicinity of the walls to a wall jet. In such case, $C_{w}$ can be derived analytically with:

$C_{w}=\frac{4 v}{d \cdot U_{\max }}$

with $U_{\max }$ the maximum velocity magnitude occurring in the vicinity of the wall, measured at a distance $d$ from this wall. However, the difficulty is that $C_{w}$ highly varies spatially along the three walls within the cavity (see Fig.4) and also varies with the aspect ratio $W / L$. In the present work, we select an averaged value of $C_{w}=0.07$ which is larger than what would be obtained by Eq. 15 at the center of the upstream wall (if $U_{\max }=U_{u}$ in Eq.15) but lower than that at the center of the downstream wall (if $U_{\max }=U_{d}$ ). Besides, as indicated by Hill (2014), a definition of the mean bottom wall friction coefficient is challenging and, in the present work, we decided to consider the same coefficient: $C_{b}=C_{w}=0.07$. This value may appear high. However, it results from a rigid body rotation with very specific velocity profiles and this value is in agreement with Hill (2014)' approach, as $C_{b}$. Hill $=5\left(u_{g} / K U\right)^{2} C_{m b} / 8=0.079$ in the present configuration for $W / L=1$. Under these assumptions, computing the four torques (Eqs. $4-10-13-14$ ) for various values of geometrical aspect ratio $W / L$ results in Fig.10 that indicates that:

- $\quad T_{i}$ continuously (almost linearly) increases with $W / L$, notably due to the continuous increase of the lever arm $l_{i}=W / 2$ (Eq.4).

- $\quad T_{w}$ increases with $W / L$ for low $W / L$ values due to an increasing wall surface and lever arm (see Eq.9) but reaches a maximum for $W / L \sim 1.5$ and slightly decreases for longer cavities due to the decrease of velocity $c$ (see Eq.8).

- $\quad T_{b}$ increases with $W / L$ due to an increase of the friction surface (equal to the ellipse horizontal surface) and the lever arm $l_{p}$ (see Eq.12); nevertheless, $T_{b}$ increasing rate rapidly decreases with $W / L$, notably due to a decrease in velocity, as mentioned just above.

- $\quad T_{p}$ becomes positive for $W / L>1$, increases with $W / L$ and reaches a maximum value for $W / L=2$ and then decreases for higher $W / L$ values. Indeed, while the lever arm of the resisting pressure torque (along the downstream wall) continuously increases with $W / L, U_{i}$ (and thus also $d P$ ) continuously decreases, see Eq.2. 
Consequently, the total resisting torque $T_{w}+T_{b}+T_{p}$ rapidly increases with $W / L$ for $0.6<W / L<1$, reaches a maximum value for $W / L \sim 2$ and slightly decreases for larger $W / L$ values, unlike the interface forcing torque that continuously increases with $W / L$. As a result, as illustrated by Fig.10, the equilibrium between resisting and forcing torques (Eq.1) is obtained for $W / L=0.7$ and 1.8 and is about valid in between $(W / L \in[0.7-1.8]$ ), considering the assumptions and simplifications used herein (such as the estimated pressure distribution along the interface and the downstream wall and the empirical constant bottom and wall frictions). Note that slight adjustments of the friction coefficients could ensure a perfect equilibrium for this range. In the end, results from Fig.10 confirm the experimental observations that a single cell is a stable flow pattern for such intermediate geometrical aspect ratio of the cavity. Oppositely, for narrower and wider cavities, Fig.10 clearly reveals that the resisting torques no more balance the forcing torque (erroneous friction coefficient values can no longer explain the torque balance discrepancies):

- For a narrow cavity $(W / L<0.6), T_{p}$ becomes a dominant forcing torque so that $T_{w}+T_{b}+T_{p}$ changes its sign. The only way to balance Eq.1 is to change the topology of the flow pattern. This is in agreement with the occurrence of the longitudinally-aligned two-cell flow pattern reported in the literature for $W / L<0.6$ and in Fig.4 for $W / L=0.3$.

- For a wide cavity $(W / L>2)$, keeping a single cell would keep the forcing torque increasing with $W / L$ while the resisting torque slowly decreases, making it impossible to balance the torque model. Fig.4 reveals that, in such case, a second cell appears and the extension (along $y$ ) of the first cell remains constant. The appearance of this second cell prevents the first cell from growing further and thus keeps the interface and resisting torques at constant magnitudes about equal to that for which the curves intersect in Fig.10 (W/L 1.8 herein). The appearance of the second cell then permits that the torque model remains balanced in the first cell even for very large $W / L$ values. The velocity gradient at the first / second cell interface only generates a new mixing layer, which itself generates a resisting torque that replaces the resisting torque imposed by the opposite wall in the one-cell flow pattern $(0.6<W / L<2)$.

\section{$\underline{3.3 \text { Identification of the parameters impacting the model results }}$}

Summarizing the model (using Eqs. 4, 10, 13 and 14) as the ratio between the resisting and interface torques leads to Eq.16:

$\frac{T_{W}+T_{b}+T_{p}}{T_{i}}=\frac{\frac{1}{2} C_{W} \frac{L}{W}\left\{\int_{-\frac{\pi}{2}+\psi}^{\frac{\pi}{2}} \cos \left[\theta-\operatorname{atan}\left(\frac{\tan (\theta)}{(W / L)^{2}}\right)\right] \mathrm{d} \theta\right\}+\frac{1}{10} C_{b} \frac{L}{h}\left\{\int_{0}^{\pi / 2} \frac{\cos \left[\theta-\operatorname{atan}\left(\frac{\tan (\theta)}{(W / L)^{2}}\right)\right]}{\sqrt{\left(\frac{W}{L}\right)^{2} \cos ^{2} \theta+\sin ^{2} \theta}} \mathrm{d} \theta\right\}+\frac{1}{8}\left(\frac{W}{L}-1\right)\left(\frac{L}{W}\right)^{3}}{0.07^{2}\left(\frac{1}{K}-\frac{L}{W}\right)^{2}}$

Besides the geometrical aspect ratio of the cavity $W / L$ (which influence on the model was discussed above, see Fig.10), this torque ratio appears to be dependent on (i) the velocity coefficient $K$, which 
equals 0.2 herein but no information is available to estimate $K$ for other geometrical and flow configurations, (ii) the bottom and wall friction coefficients $C_{b}$ and $C_{w}$ which were taken constant and equal to 0.07 herein while their magnitudes for other configurations remains unknown, (iii) the shallowness parameter $L / h$ which impact on the model is now discussed. Keeping $K=0.02$ and $C_{b}=C_{w}=0.07$, Fig. 11 plots the evolution of the torque ratio (Eq.16) with $W / L$ for the eight shallowness values $L / h$ reported by the literature review (see Table 1 ). Fig. 11 reveals that as $L / h$ increases, the range of geometrical aspect ratio over which the torque ratio is fairly balanced (i.e., equal or slightly larger than 1) increases from $W / L \in[0.86 ; 1.36]$ for $L / h=0.9$ to $W / L \in[0.59 ; 2.97]$ for $L / h=15$. It is then expected that for shallower configurations (larger $L / h$ values): (i) the transition from a flow pattern with 2 cells aligned longitudinally (noted " $2 \mathrm{~L}$ " in Table 1 ), to a flow pattern with a single cell (noted " 1 " in Table 1), occurs for a lower $W / L$ value; and (ii) the transition from a single cell to two cells aligned transversally (noted " 2 " " in Table 1), occurs for a larger $W / L$ value. In other words, the single cell configuration is expected to occur for a larger range of geometrical aspect ratios $W / L$ for shallow than for deep configurations.

\subsection{Application of the model to the literature data}

Eq.16 is now applied to the configurations previously measured in the literature (listed in Table 1), using the $W / L$ and $L / h$ ratio values reported by the authors (when available) and the parameters $K=0.02$ and $C_{b}=C_{w}=0.07$ obtained above for the present configuration. The flow patterns predicted by the model is indicated as symbols in Fig. 11 and in the last column of Table 1 and should be compared to the reported flow patterns indicated in the $6^{\text {th }}$ column.

It is noted in Table 1 that the model cannot be applied to very narrow cavities, such as Nezu \& Onitsuka (2002a \& b) configurations with $W / L=0.2-0.33$. Indeed, Mignot et al. (2017) (in their figure 10 and their equation 10) indicate that $\delta$, the width of the mixing layer width at the cavity/main stream interface, linearly increases along $x$ axis as $\mathrm{d} \delta / \mathrm{d} x \sim 0.2$. The half width of the mixing layer when reaching the downstream wall thus equals about $0.1 L$, i.e. about $0.4 \mathrm{~W}$ if $W / L \sim 0.25$. In other words, the mixing layer occupies almost half of the cavity width in the downstream wall region and thus strongly interacts with the recirculation cell in a complex way that is not considered by the torque model: the mixing layer is expected to be strongly affected by the recirculation cell, so that $T_{i}$ (Eq.4) is no more valid and the flow entrainment in the cavity is also expected to be strongly affected. These configurations are thus quoted as "not applicable" of the model in Table 1.

For the other configurations, the model appears to fairly predict all reported flow patterns (" 2 L", " 1 " and " $2 \mathrm{~T}$ " patterns) except for one configuration from Mizumura and Yamasaka (2002) for which the model predicts a " $2 \mathrm{~L}$ " pattern while the authors reported a " 1 " cell pattern; no clear explanation for this discrepancy could be encountered. To conclude, the model (Eq.16) is able to predict the number of cells in all rectangular cavity flow, as long as the aspect ratio exceeds $W / L=0.33$. 


\section{Temporal stability and turbulent characteristics}

\subsection{Temporal stability of the velocity fields}

All velocity fields plotted and computed in the previous sections were time-averaged and the aim of the present section is to evaluate the temporal fluctuations of these flows. To do so, Fig.12 shows for two configurations ( $W / L=1$ and 3$)$ the flow field timely-averaged over five increasing durations $(2,5$, 10, 20 and 200s). It appears that 2 seconds (i.e. 24 samples) of measurement is enough to obtain a fair representation of the velocity field, even though the details of the flow pattern differ from the fully converged mean velocity field (200s, using 2440 samples). Oppositely, when considering a measurement duration of $5 \mathrm{~s}$ (61 samples) and all the more 10s (122 samples), the mean flow field becomes very similar to the fully converged flow field. Two reasons for the differences of flow pattern between the 2 s (24 samples) and the fully converged flow can be put forward: either i) the use of only 24 samples is not sufficient to overcome the measurement uncertainties or ii) physical fluctuations of the velocity fields exist at frequencies of order of magnitude equal to about $1 \mathrm{~Hz}$, so that an averaging over only 2 seconds of physical time is not sufficient. To identify the correct reason, the last plot in Fig.12a and Fig.12b (within boxes) is averaged over a long duration (200s) but with a very low number of samples: only 12 samples. For both aspect ratios, the plots exhibit high similarities with the fully converged velocity field, proving that averaging over only 12 samples is sufficient to cancel most measurement uncertainties. Going back to the 2 seconds duration velocity field averaged over 24 samples (that is twice as many samples as the previously discussed velocity field) the discrepancies when compared to the converged velocity field should finally be due to relatively low frequency motions (of order of magnitude $1 \mathrm{~Hz}$ ). These low frequency motions are more deeply analyzed in the sequel.

\section{$\underline{4.2 \text { Turbulent characteristics }}$}

Fig.13a and $13 \mathrm{~b}$ reveal that the low frequency motions responsible for the relatively long convergence time of the mean velocity field are actually related to the advection of coherent turbulent structures within the cavity. Mignot et al. (2016) previously showed that turbulent structures are periodically shed from the region surrounding the upstream corner of the cavity and are advected along the interface towards the downstream corner with a peak frequency equal to $0.6 \mathrm{~Hz}$ for the $W / L=1$ configuration. Moreover, these authors showed that as the turbulent structures reach the downstream corner, they either end up in the main stream or enter the cavity. Fig.13a reveals that the structures entering the cavity near the downstream corner are advected within the outer layer of the recirculation cell, along the downstream wall and then along the opposite wall. These turbulent structures thus follow the cavity mean-flow streamlines (plotted in the first graph of Figs.13a and 13b). As they reach the upstream/opposite corner, the structures lose much energy but their trajectory can extend all the way towards the upstream corner of the interface (as for structure $\mathrm{C}$ ). The turbulent structures can also split $\left(A_{1}\right.$ and $\left.A_{2}\right)$, merge $\left(A_{1}\right.$ and $\left.D\right)$, or reach the inner layer of the recirculation cell (B). Moreover, 
Fig. $13 \mathrm{~b}$ reveals that for a configuration with two cells $(W / L=3)$, some coherent structures even succeed in passing from the first to the second cell (as for structure F).

The resulting spatial distribution of turbulent intensity in the cavity is plotted in Fig.14 for cavities with $W / L=1$ and 3 . This figure confirms that the maximum turbulent activity takes place in the outer layer of the first cell: it is maximum along the cavity / main stream interface and then gradually decreases along the downstream wall, the opposite limit of the first cell and finally along the upstream wall, as already noticed in Fig.13. An interesting feature appears in Fig.14b: within the second cell, a local maximum intensity is measured along the opposite wall; Fig.13b indeed reveals that turbulent structures seem to remain in this region for long times.

Mignot et al. (2016) showed that, while the turbulent structures shedding along the interface is periodic, the entrance of structures into the cavity is not periodic (see figure 10 of these authors) so that the fluctuating motions in the cavity are not periodic. Turbulent spectra of velocity along the downstream wall (not shown here) do not reveal any peak frequency. To conclude, the turbulent structures entering the cavity generate aperiodic and complex time fluctuations of the velocity field, which confirms that an averaging time of a few seconds (5 to 10 seconds in Fig.12) is required to reach a fully converged velocity field.

\section{Conclusions}

The present paper aimed at characterizing the flow patterns forming in lateral, rectangular cavities connected to a main (river) stream, focusing on the transition between these flow patterns. Compared to the existing literature, the experimental work took benefit of a devoted facility allowing to vary continuously the aspect ratio and to reach unexplored (at least to the authors knowledge in openchannel flows) values reaching $W / L=5$. Velocity measurements were performed in flows where the characteristics of the main stream were kept constant while the width of the lateral cavity was regularly increased. The flow patterns previously reported in the literature were retrieved, namely: for a quite narrow cavity, two cells are aligned along the mean flow axis; for a close-to-square cavity, one cell occupies the whole available cavity volume; and finally for a wide cavity, two cells are aligned in the direction perpendicular to the mean flow axis. It also appeared that as long as a single cell is present in the cavity (i.e., for a geometrical aspect ratio of $1<\mathrm{W} / \mathrm{L} \leq 2$ ), the profile of velocity magnitude along the streamwise axis of the cavity remains unchanged (with a constant maximum velocity magnitude near the upstream and downstream walls, equal to $K U$ with $K=0.2$ in the present flow cases), so that the entrained discharge recirculating in the cavity also remains equal.

In order to gain information on the transition between the flow patterns, a torque model was proposed and applied to the flow in the cavity, extending the approach previously introduced by Hill (2014) for squared cavities $(W / L=1)$ to rectangular geometries. When applied to the present experimental 
configurations, the model, even if highly simplified, proves to fairly predict all reported flow patterns and thus also the corresponding transitions (except for very narrow cavities). The model's results reveal that the transition from the one-cell to the two-cells flow patterns (for low and high $W / L$ values) is required to maintain the torque balance in the cavity satisfied. Besides, when non-dimensionalized, the torque model (Eq.16) indicates that the parameters affecting the transitions between the three flow patterns are: the velocity coefficient $K$, the bottom and wall roughness coefficients $C_{b}$ and $C_{w}$ (which are expected to vary with the Reynolds number of the cavity and thus with the Reynolds number of the main stream) and the shallowness parameter $L / h$. Assuming constant values for $K, C_{b}$ and $C_{w}$, the model predicts that the range of $W / L$ for which the single cell pattern remains stable increases with $L / h$. The model finally proves to be able to predict the flow patterns from all configurations from the literature except for one single flow configuration.

Finally, the experimental work showed that the velocity fields in the cavity are quite stable in time. Indeed, a time-averaging over five to ten seconds appears sufficient to reach a converged mean flow pattern even if intermediate frequency motions of the flow field occur. The origin of these oscillations of typical frequency of about $1 \mathrm{~Hz}$ are due to the non-periodic shedding of turbulent structures from the mixing layer towards the cavity and their advection all along the recirculation cells of the cavity.

Apart from the scalar dispersion, the present results have major applications for the geomorphology of dead zones. The high spatial variability of velocity magnitude in the cavity, within each cell and between the adjacent cells, should lead to a highly variable spatial distribution of deposition and thus to complex bed forms on the bottom of the cavity that may, in turn, impact its hydrodynamics. Moreover, it would be interesting to relate the time required to obtain a stable bed morphology in the cavity to the typical annual flood/drought time cycles. Also, if the bed and/or suspended sediment is poly-dispersed, some complex particle size sorting should additionally occur and this would have important application in river ecology.

\section{References}

Argerich, A., Marti, E., Sabater, F., Haggerty, R., Ribot, M. (2011). "Influence of transient storage on stream nutrient uptake based on substrata manipulation.” Aquatic Sciences. 73, 365-376.

Booij, R. (1989). "Exchange of mass in harbors." $23^{\text {rd }}$ congress of IAHR, 21-25 August 1989, Ottawa, Canada, D69-D74.

Cheng, M., and Hung K. C. (2006). "Vortex structure of steady flow in a rectangular cavity." Computers \& Fluids. 35, 1046-1062.

Douay, C. L., Pastur, L. R., and Lusseyran F. (2016). "Centrifugal instabilities in an experimental open cavity flow." Journal of Fluid Mechanics. 788, 670-694. 
Ensign, S. H., Doyle, M. W. (2005) "In-channel transient storage and associated nutrient retention: evidence from experimental manipulations“. Limnology and Oceanography. 50(6), 1740-1751.

Faure T.M., Adrianos P., Lusseyran F., Pastur L. (2007). "Visualizations of the flow inside an open cavity at medium range Reynolds numbers." Experiments in Fluids. 42 (2), 169-184.

Hill, D.F. (2014). "Simple model for the recirculation velocity of open-channel embayments." Journal of Hydraulic Engineering. 140(4), 06014004.

Hinterberger, C., Fröhlich, J., and Rodi W. (2007). "Three-Dimensional and Depth-Averaged LargeEddy Simulations of Some Shallow Water Flows.” Journal of Hydraulic Engineering. 133(8), 857872.

Jackson, T.R., Apte, S.V., Haggerty, R., and Budwig, R. (2015). "Flow structure and mean residence times of lateral cavities in open channel flows: influence of bed roughness and shape." Environmental Fluid Mechanics. 15(5), 1069-1100

Jahanmiri, M. (2011). "Particle image velocimetry: Fundamentals and its application." in Report 2011:03, ISSN 1652-8549, Chalmers University of Technology, Goteborg, Sweden.

Kimura, I. and Hosoda, T. (1997). "Fundamental properties of flows in open channels with dead zone.” Journal of Hydraulic Engineering. 123(2), 98-107.

Koseff, J.R. and Street, R.L. (1984). "The lid-driven cavity flow: a synthesis of qualitative and quantitative observations." Journal of Fluids Engineering ASME, 106, 3, 390-398.

Le Coz, J., Brevis, W., Niño, Y., Paquier, A., and Rivière, N. (2006). "Open-channel side-cavities: a comparison of field and flume experiments." River Flow 2006, vol 1, 145-152, Lisbon, Portugal, Sept. 6-8, 2006.

Li, C.W. and Ip, K.W. (1999). "Residence time in semi-enclosed water bodies." Civil and Environmental Engineering Conference, 8-12 November 1999, Bangkok, Thailand, VI.13-VI.16

Lin L.S., Chen Y.G., Lin C.A. (2011). "Multi relaxation time lattice Boltzmann simulations of deep lid driven cavity flows at different aspect ratios." Computers and Fluids. 45, 233-240.

McCoy, A., Constantinescu, G., and Weber, L.J. (2008). "Numerical investigation of flow hydrodynamics in a channel with a series of groynes." Journal of Hydraulic Engineering. 134(2), $157-172$

Mignot, E., Doppler, D., Riviere, N., Vinkovic, I., Gence, J., and Simoens, S. (2014). "Analysis of flow separation using a local frame-axis: application to the open-channel bifurcation." Journal of Hydraulic Engineering. 140, 280-290.

Mignot, E., Cai, W., Launay, G., Riviere, N., and Escauriaza, C. (2016). "Coherent turbulent structures at the mixing-interface of a square open-channel lateral cavity." Physics of Fluids. 28, 045104

Mignot, E., Cai, W., Polanco, J.I., Escauriaza, C., and Riviere, N. (2017). "Measurement of mass exchange processes and coefficients in a simplified open-channel lateral cavity connected to a main stream." Environmental Fluid Mechanics, 17 (3), 429-448. 
Mizumura, K. and Yamasaka, M. (2002). "Flow in open channel embayments." Journal of Hydraulic Engineering. 128(12), 1098-1101.

Muto, Y., Imamoto, H. and Ishigaki, T. (2000). "Velocity measurements in a straight open channel with a rectangular embayment." Proc. $12^{\text {th }}$ APD-IAHR, Bangkok, Thailand, 353.

Nezu I. and Onitsuka, K. (2002a). "LDA measurements of side-cavity open-channel flows - Wando models in rivers." Fluid modeling and turbulence measurements (eds. Ninokata et al.), World Scientific, 169-176.

Nezu, I. and Onitsuka, K. (2002b). "PIV measurements of side-cavity open-channel flows - Wando models in rivers." Journal of Visualization. 5(1), 77-84.

Peng, Y. F., Shiau, Y. H., Hwang, R. R. (2003). "Transition in a 2-D lid-driven cavity flow." Computers and Fluids. 32, 337-352.

Rodi,W. (1993). Turbulence models and their application in hydraulics-A state of the art review, 3rd Ed., IAHR, Delft, Netherlands.

Sanjou, M. and Nezu, I. (2013). "Hydrodynamic characteristics and related mass-transfer properties in open-channel flows with rectangular embayment zone." Environmental Fluid Mechanics. 13(6), $527-555$.

Sanjou, M., Akimoto, T., and Okamoto, T. (2012). "Three-dimensional turbulence structure of rectangular side-cavity zone in open-channel streams." International Journal of River Basin Management. 10(4), 293-305.

Shankar P.N., and Deshpande M.D. (2000). "Fluid mechanics in the driven cavity". Annual Review of Fluid Mechanics, 32, 93.

Taneda S. (1979). "Visualization of separating Stokes flows". Journal of the Physical Society of Japan, 46, 1935.

Tominaga, A. and Jong, J. (2011). "Effects of configuration on flow structures and sediment deposition in a concavity zone of flood plain." 31th IAHR World Congress - Balance and Uncertainty. June $26^{\text {th }}$-July $1^{\text {st }}$, Brisbane, Australia.

Tuna, B.A., Tinar, E., and Rockwell, D. (2013). "Shallow flow past a cavity: globally coupled oscillations as a function of depth.” Experiments in Fluids. 54, 1586.

Uijtewaal, W., Lehmann, D., and van Mazijk, A. (2001). "Exchange processes between a river and its groyne fields: model experiments." Journal of Hydraulic Engineering. 127(11), 928-936.

Weitbrecht, V., Socolofsky, S.A., and Jirka, G.H. (2008). "Experiments on mass exchange between groin fields and main stream rivers." Journal of Hydraulic Engineering. 134(2), 173-183.

Younis, M. Y., Zhang, H., Hu, B., and Mehmood, S. (2014). "Topological evolution of laminar juncture flows under different critical parameters." Science China, Technological Sciences. 57(7), 1342-1351. 


\begin{tabular}{|c|c|c|c|c|c|c|}
\hline Id & Authors & $W / L$ & $L / h$ & $\begin{array}{c}\text { Method } \\
\text { (Computed } \\
\text { or } \\
\text { Measured) } \\
\end{array}$ & $\begin{array}{l}\text { Number of } \\
\text { measured cells }\end{array}$ & 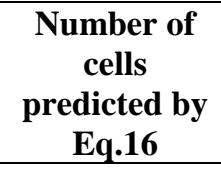 \\
\hline \multicolumn{7}{|c|}{ Lateral cavities } \\
\hline Bo89 & $\begin{array}{l}\text { Booij } \\
(1989)\end{array}$ & $1 \& 3$ & 9.1 & M & $1 \& 2_{\mathrm{T}}$ & $1 \& 2_{\mathrm{T}}$ \\
\hline \multirow[t]{3}{*}{ Ki97 } & $\begin{array}{c}\text { Kimura \& Hosoda } \\
\text { (1997) }\end{array}$ & $0.66 \& 1$ & $11.1 \& 15$ & $\mathrm{C}$ & $1 \& 1$ & $1 \& 1$ \\
\hline & $\begin{array}{l}\text { Muto et al. } \\
\quad(2000)\end{array}$ & $0.33 \& 1$ & Unknown & M & $2_{\mathrm{L}} \& 1$ & Lack of data ${ }^{(2)}$ \\
\hline & $\begin{array}{l}\text { Nezu \& Onitsuka } \\
\quad(2002 \mathrm{a} \& \text { b) }\end{array}$ & $0.2 \& 0.33$ & $5 \& 3.8$ & M & $1 \& 1$ & $\begin{array}{c}\text { Not } \\
\text { applicable }^{(3)}\end{array}$ \\
\hline $\mathrm{Mi02}$ & $\begin{array}{c}\text { Mizumura \& } \\
\text { Yamasaka (2002) }\end{array}$ & $0.5 \& 1$ & $3.6 \& 1.8$ & $\mathrm{M} \& \mathrm{C}$ & $1 \& 1$ & $2 \mathrm{~L} \& 1$ \\
\hline \multirow[t]{2}{*}{ Le06 } & $\begin{array}{l}\text { LeCoz et al. } \\
\quad(2006)\end{array}$ & 0.5 & 0.9 & M & $2 \mathrm{~L}$ & $22_{L}$ \\
\hline & $\begin{array}{c}\text { Tominaga \& Jong } \\
\text { (2011) }\end{array}$ & 0.5 & 8.6 & M & $?^{(1)}$ & $2 \mathrm{~L}$ \\
\hline Sa12 & $\begin{array}{l}\text { Sanjou et al. } \\
\quad(2012)\end{array}$ & 0.33 & 6 & $\mathrm{C}$ & $2 \mathrm{~L}$ & $2 \mathrm{~L}$ \\
\hline Sa13 & $\begin{array}{c}\text { Sanjou \& Nezu } \\
(2013)\end{array}$ & 0.33 & 5.7 & M & $2 \mathrm{~L}$ & $2 \mathrm{~L}$ \\
\hline \multicolumn{7}{|c|}{ Cavities between two groynes } \\
\hline & $\begin{array}{l}\text { Uijttewaal et al. } \\
\text { (2001) }\end{array}$ & $\begin{array}{l}0.3 \\
0.7\end{array}$ & & $\begin{array}{c}2 \mathrm{~L} \\
1\end{array}$ & M & \\
\hline & $\begin{array}{l}\text { Hinterberg et al. } \\
\text { (2007) }\end{array}$ & 0.4 & & $2 \mathrm{~L}$ & $\mathrm{C}$ & \\
\hline & $\begin{array}{l}\text { Weitbrecht } \\
\text { et al. } \\
(2008)\end{array}$ & $\begin{array}{c}0.35-0.59 \\
0.77 \& \\
1.12 \\
2 \& 3.35\end{array}$ & & $\begin{array}{c}2_{\mathrm{L}} \\
1 \\
2_{\mathrm{T}}\end{array}$ & M & \\
\hline & $\begin{array}{l}\text { McCoy et al. } \\
(2008)\end{array}$ & 0.7 & & 1 & $\mathrm{C}$ & \\
\hline
\end{tabular}

Table 1: Application of the torque model to the simplified isolated rectangular lateral cavity flows available in the literature along with a literature review of the flow patterns in cavities located between two groynes. Number of cells " $2_{L}$ " refers to 2 cells aligned longitudinally (along $x$ axis) and " $2_{T}$ " to 2 cells aligned transversally (along y axis).

${ }^{(1)}$ Tominaga \& Jong (2011) measured their velocity field very close to the bottom where the flow is known to vary with regards to the quasi-2D flow and the authors report a large "stagnant area" upstream and a very asymmetrical downstream vortex; it is then not so clear whether one or two cells is present away from the bottom region. ${ }^{(2)}$ The water depth considered by Muto et al (2000) remains unknown. ${ }^{(3)}$ The model (Eq.16) cannot be applied to cavity aspect ratios as low as those used by Nezu \& Onitsuka $(2002 a \& b)$, see explanations in the text. 

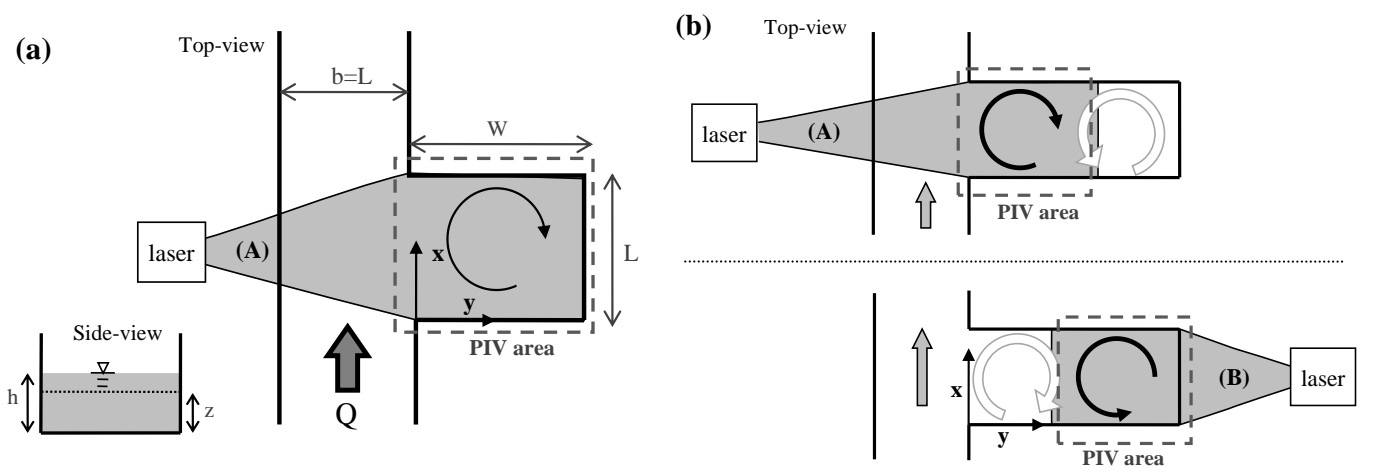

Figure 1: Sketch of the PIV image acquisition methods for narrow cavities $(W / L<2.3$, a) and wider cavities $(W / L>2.6, b)$ 


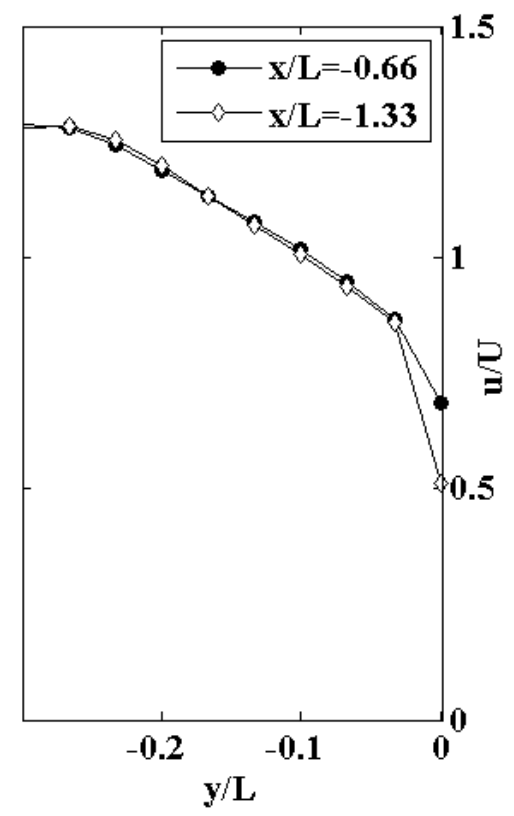

Figure $2:$ Lateral boundary layer attached to the right lateral wall in the inlet channel at $z / L=0.71$ measured for the $W / L=1$ cavity configuration 

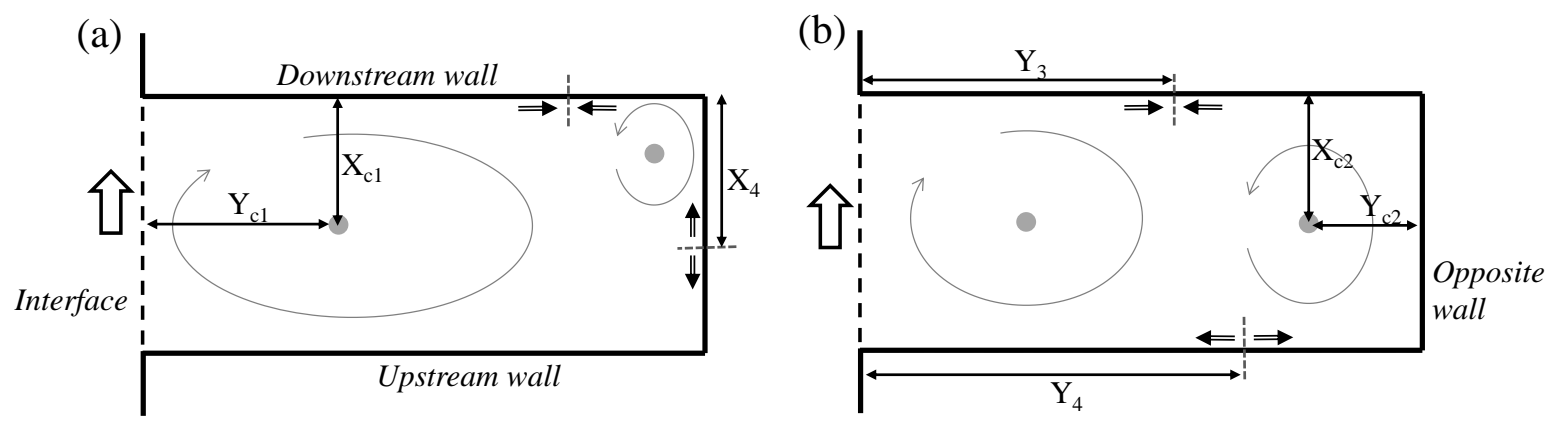

Figure 3. Definition of the geometrical parameters, sketched over a cavity with a small confined secondary cell (a) and with a large secondary cell occupying the whole cavity width (b). The dash lines with a pair of arrows represent the separation and reattachment points. 

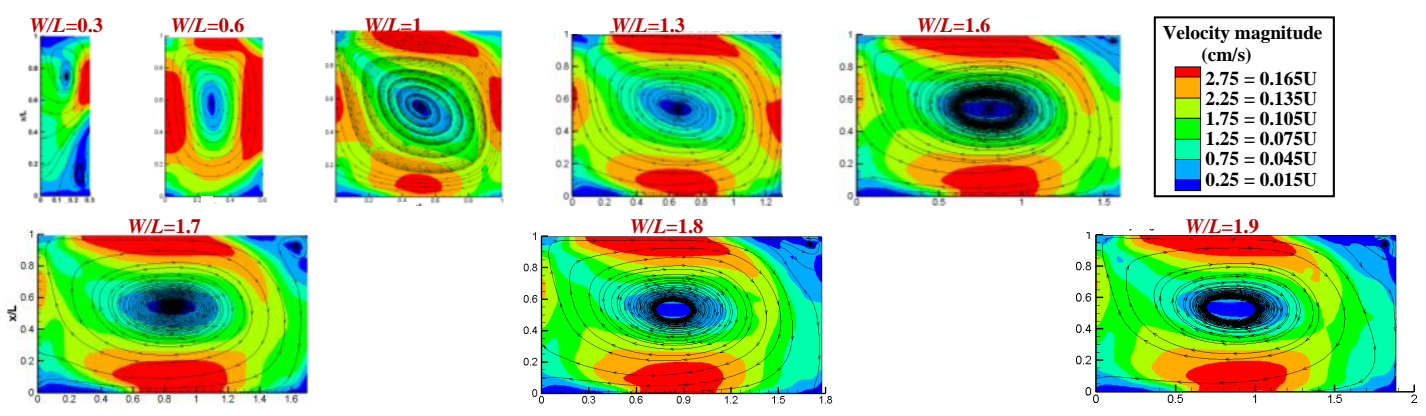

$W / L=$

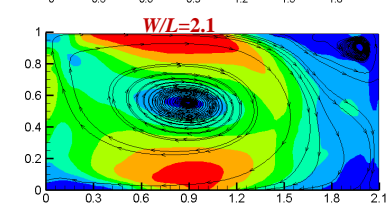

$W / L=2.2$
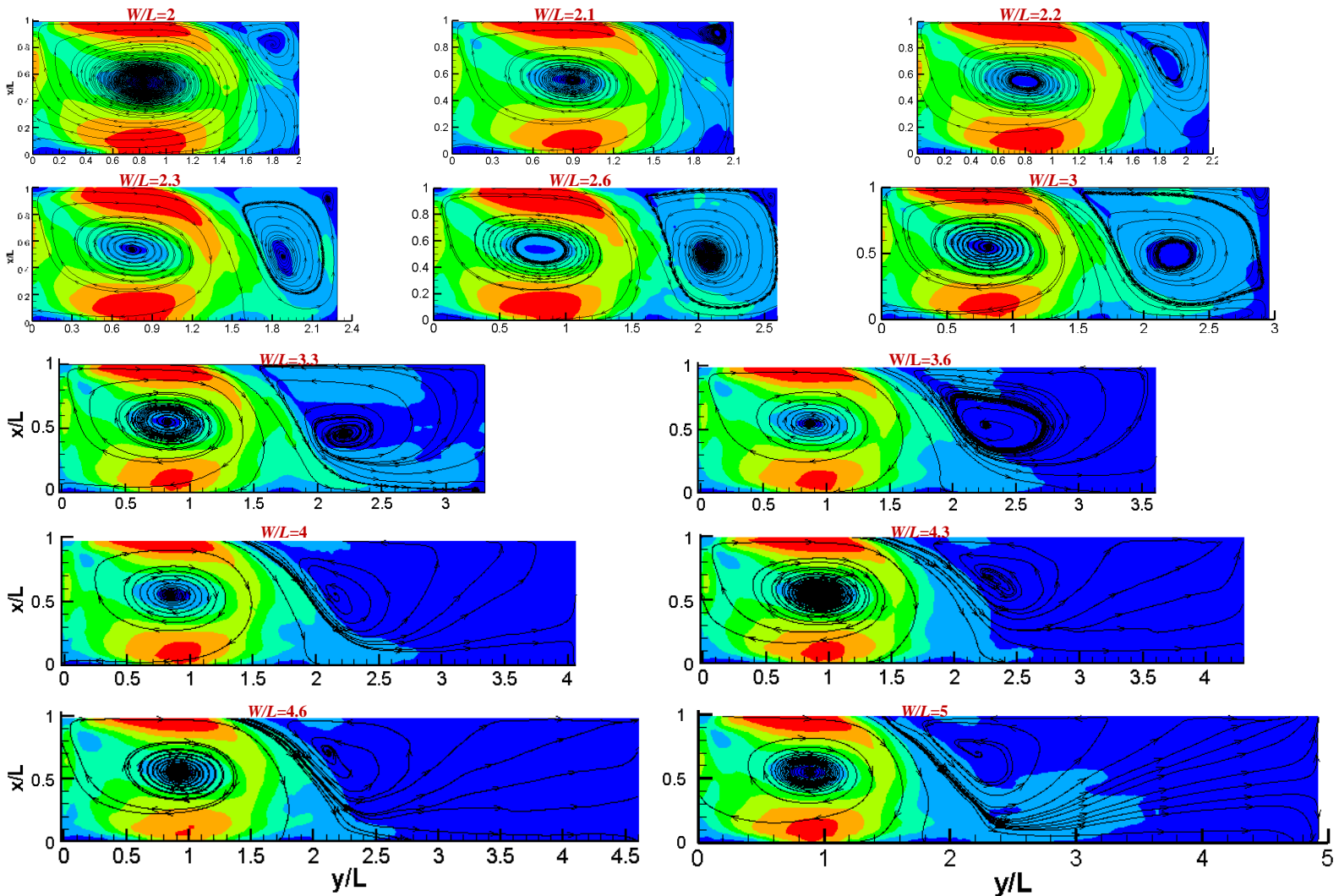

Figure 4. $2 D$ fields of velocity magnitudes measured at $z / L=0.71$ for 20 different aspect ratios of the cavity $W / L$. 

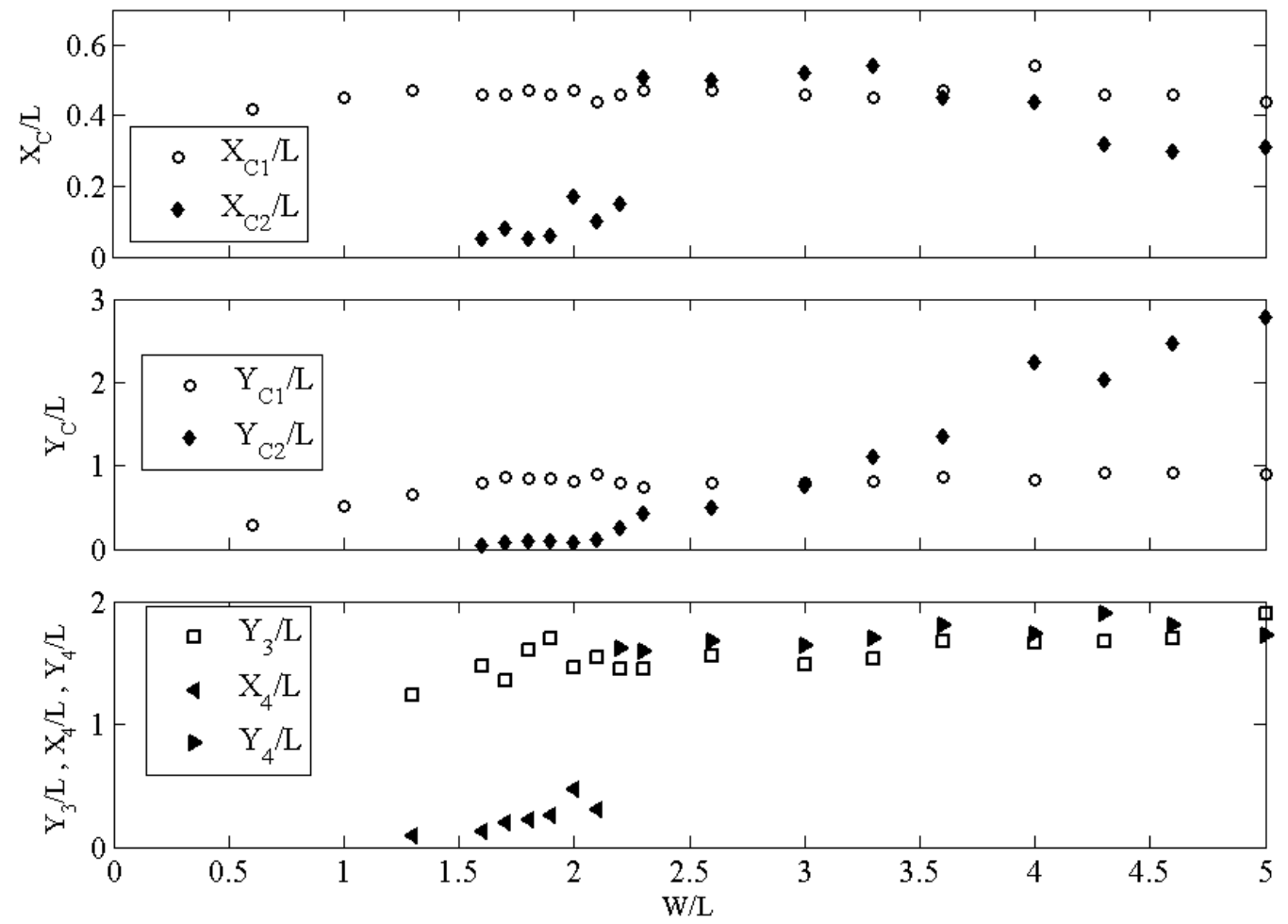

Figure 5: Evolution of the geometrical parameters estimated at $z / h=0.71$ for increasing $W / L$ aspect ratios. 

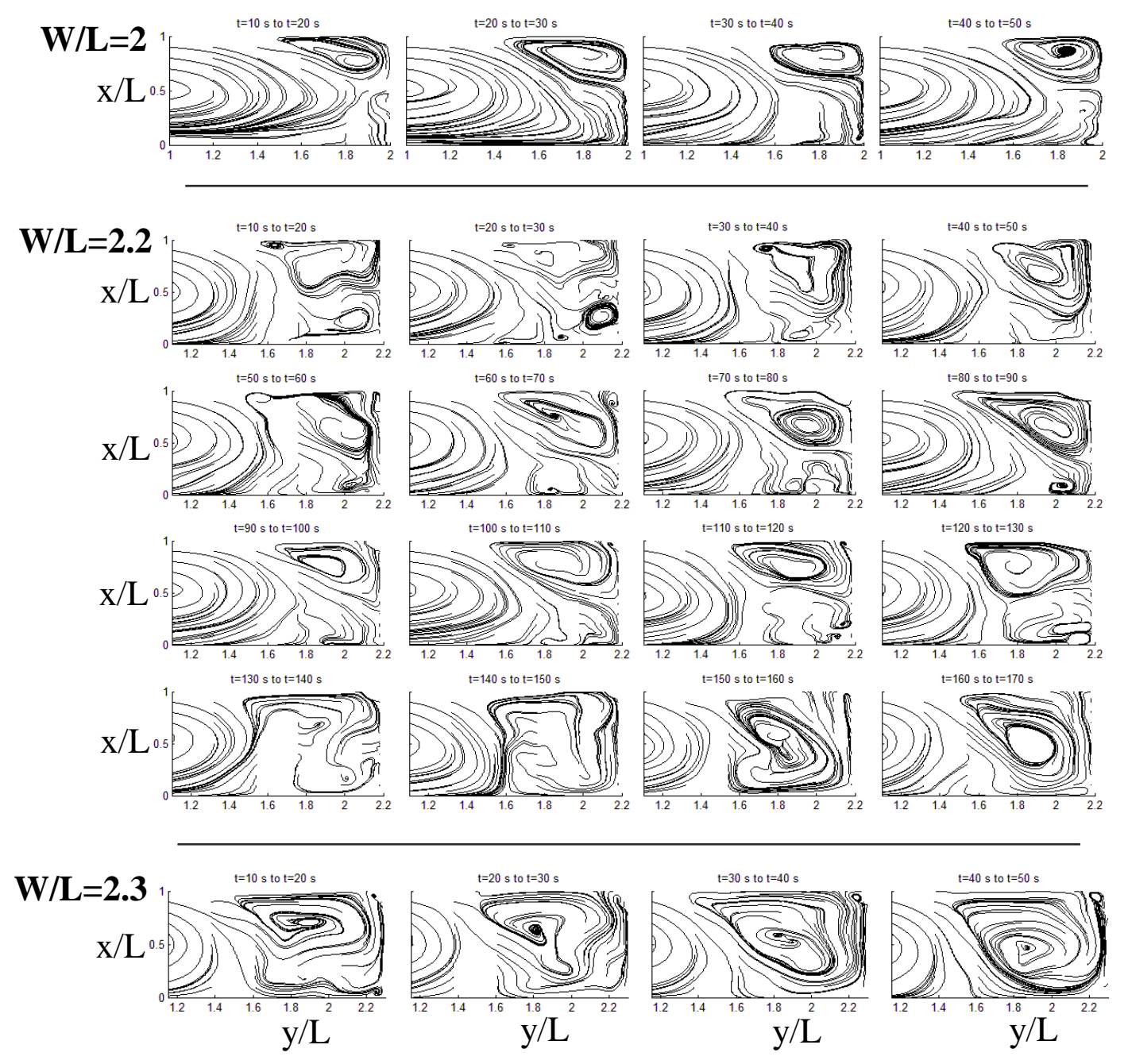

Figure 6. Streamlines of the flow at $z / h=0.71$ averaged over 10 seconds, and plotted every 10 seconds for $W / L=2,2.2 \& 2.3$. 

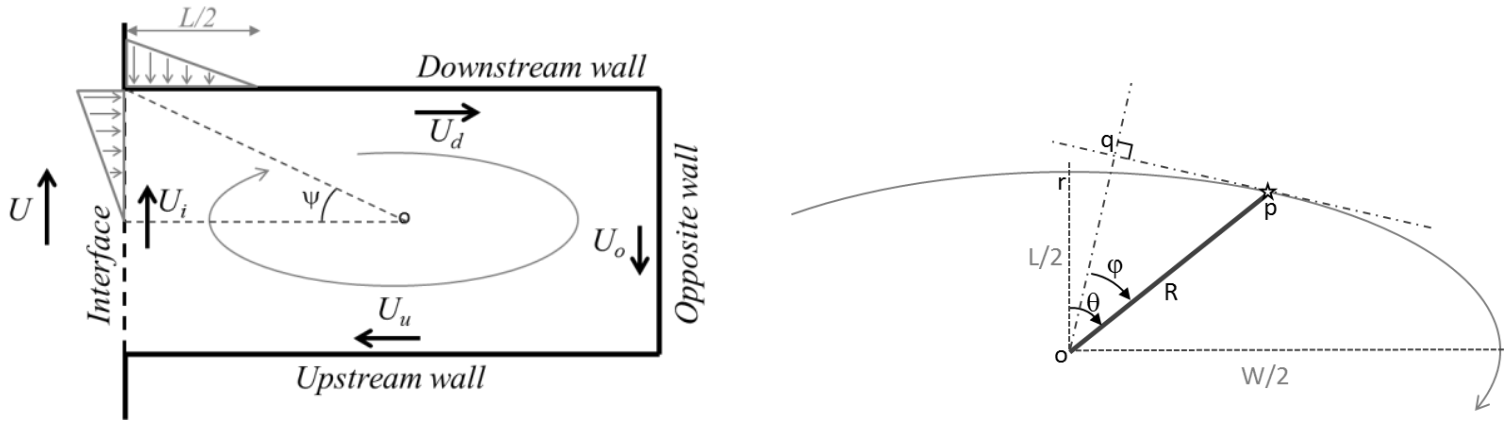

Figure 7. Scheme of the cavity including parameters used in the equilibrium torque balance model 

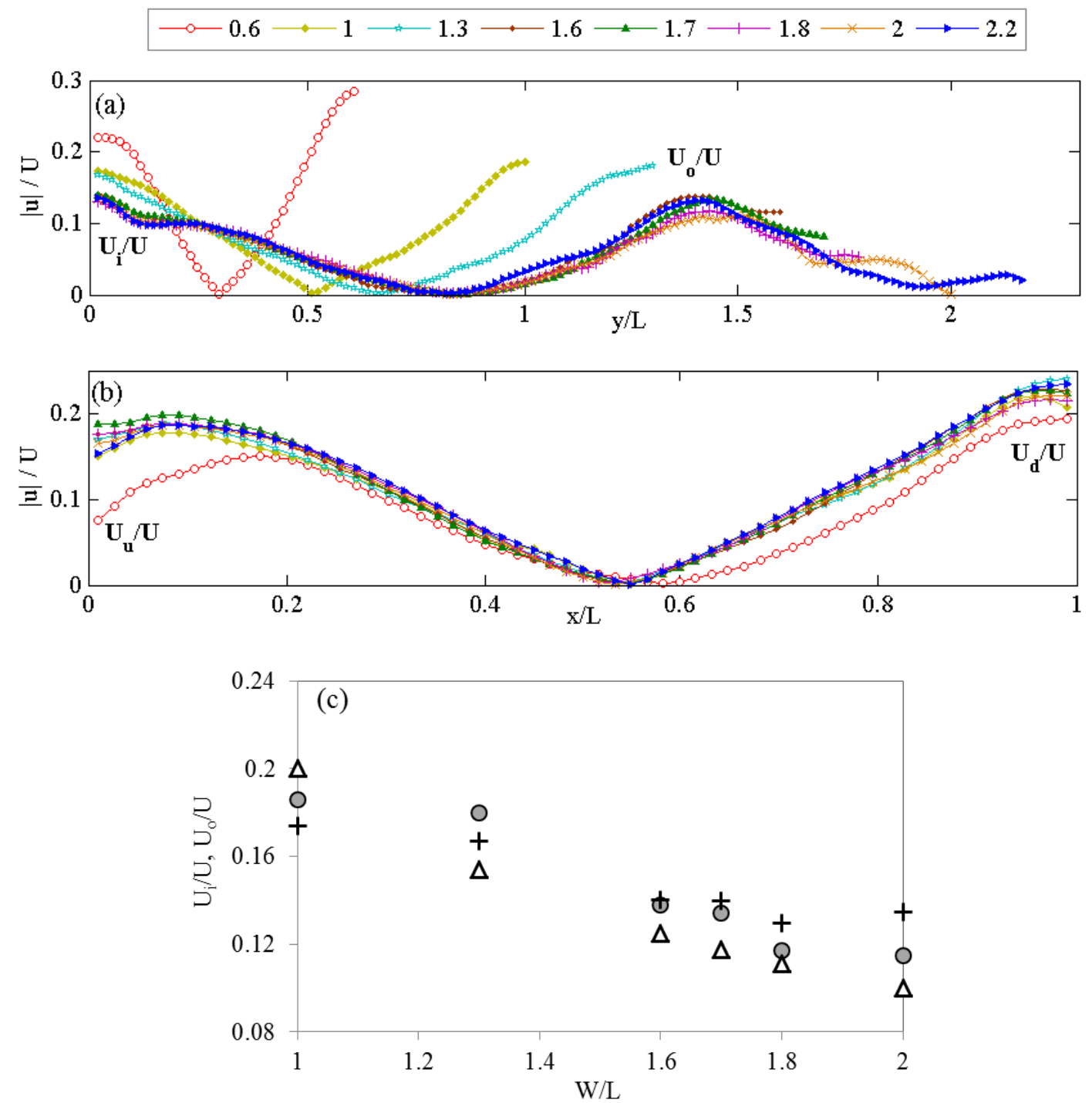

Figure 8. Profiles of mean velocity magnitude along y axis (a) and $x$ axis (b) passing through the center of the first cell (as defined in Figure 3) for a selection of 8 cavities with increasing aspect ratios. $U_{i}, U_{o}, U_{u}$ and $U_{d}$ are the maximum velocity magnitudes measured near the centers of, respectively, interface, opposite, upstream and downstream walls. (c): Maximum measured interface $\left(U_{i} / U,+\right)$ and opposite $\left(U_{o} / U, \bullet\right)$ velocities along with the predicted non-dimensional velocity $0.2 L / W$ $(\Delta)$. Note that due to the large measurement domain and too low spatial resolution $(5 \mathrm{~mm})$, the velocity decrease to 0 at the walls (no-slip conditions) could not be captured herein. 

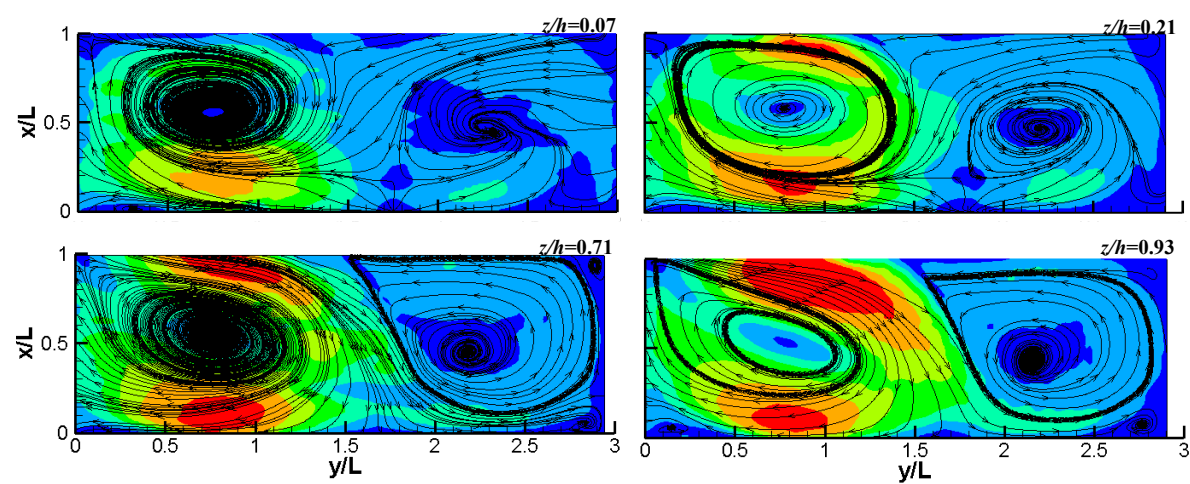

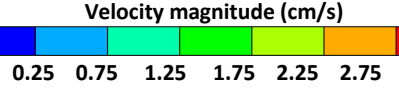

Figure 9. 2D mean velocity fields measured along four elevations for the configuration $W / L=3$. 


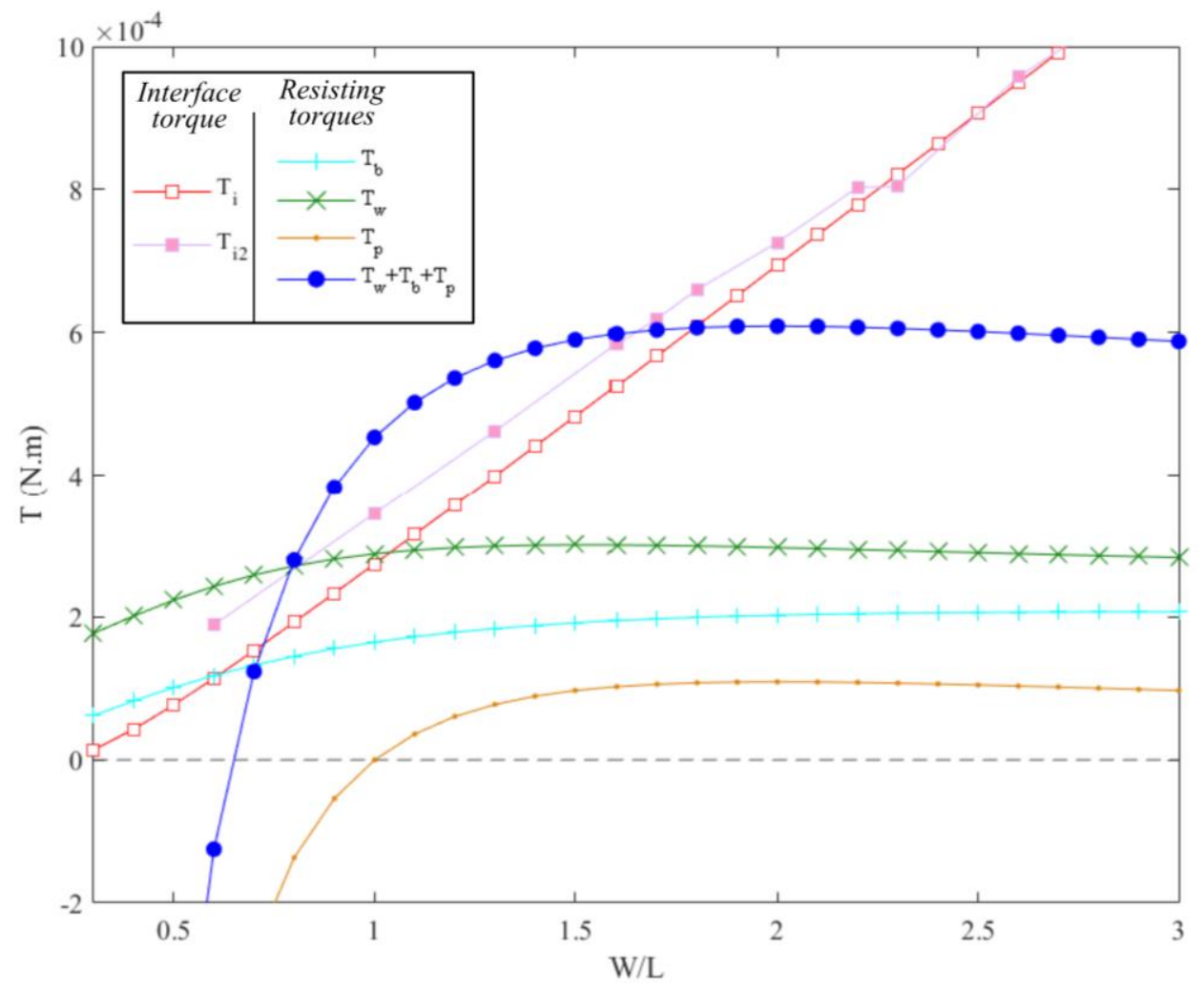

Figure 10. Results of the equilibrium torque balance model (Eq.1): evolution with the geometrical aspect ratio of the cavity $W / L$ of all torque terms. 


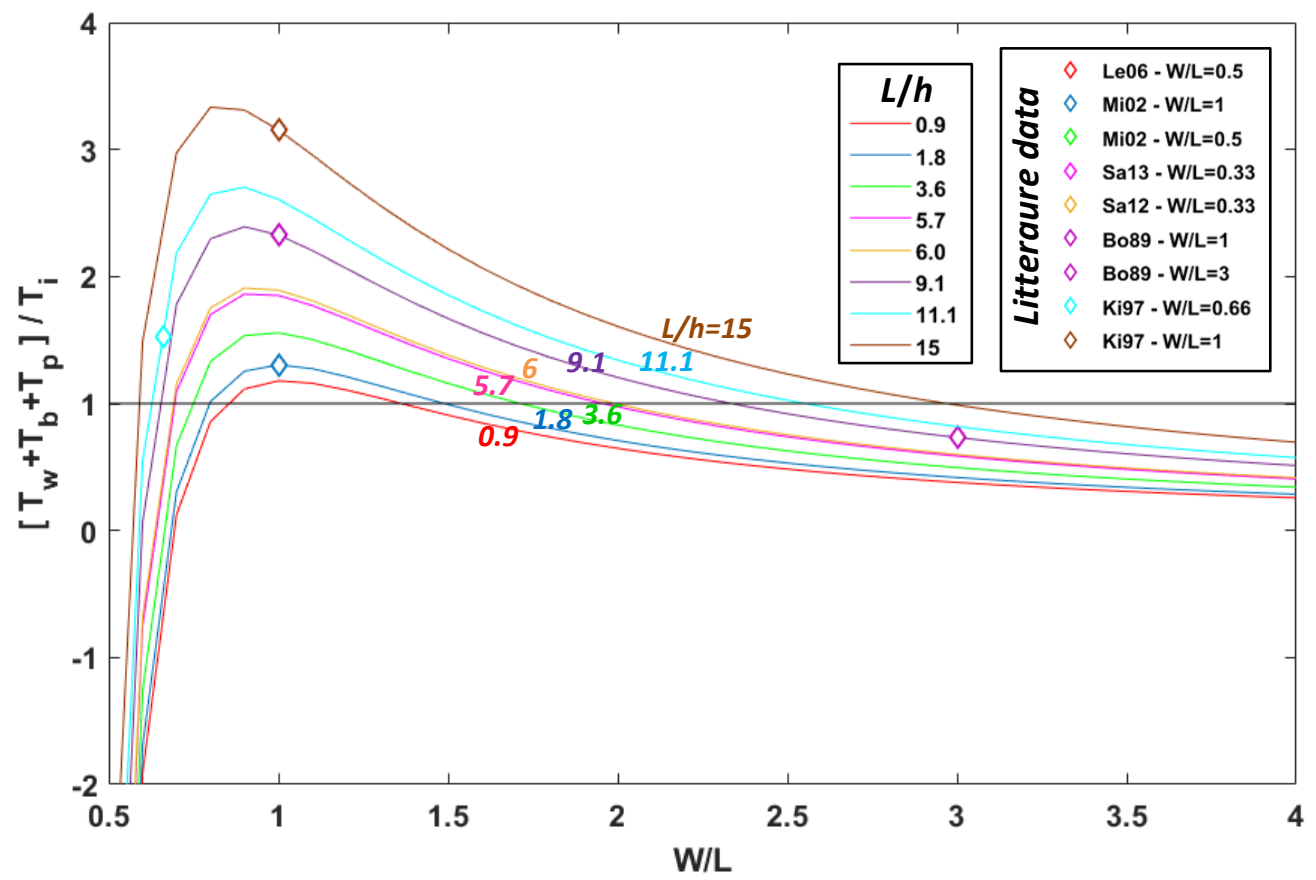

Figure 11. Evolution of the resistance over forcing torque ratio (Eq.16) with the geometrical aspect ratio of the cavity for the eight shallowness values available in the literature, along with the model prediction for the literature data, as reported in Table 1 

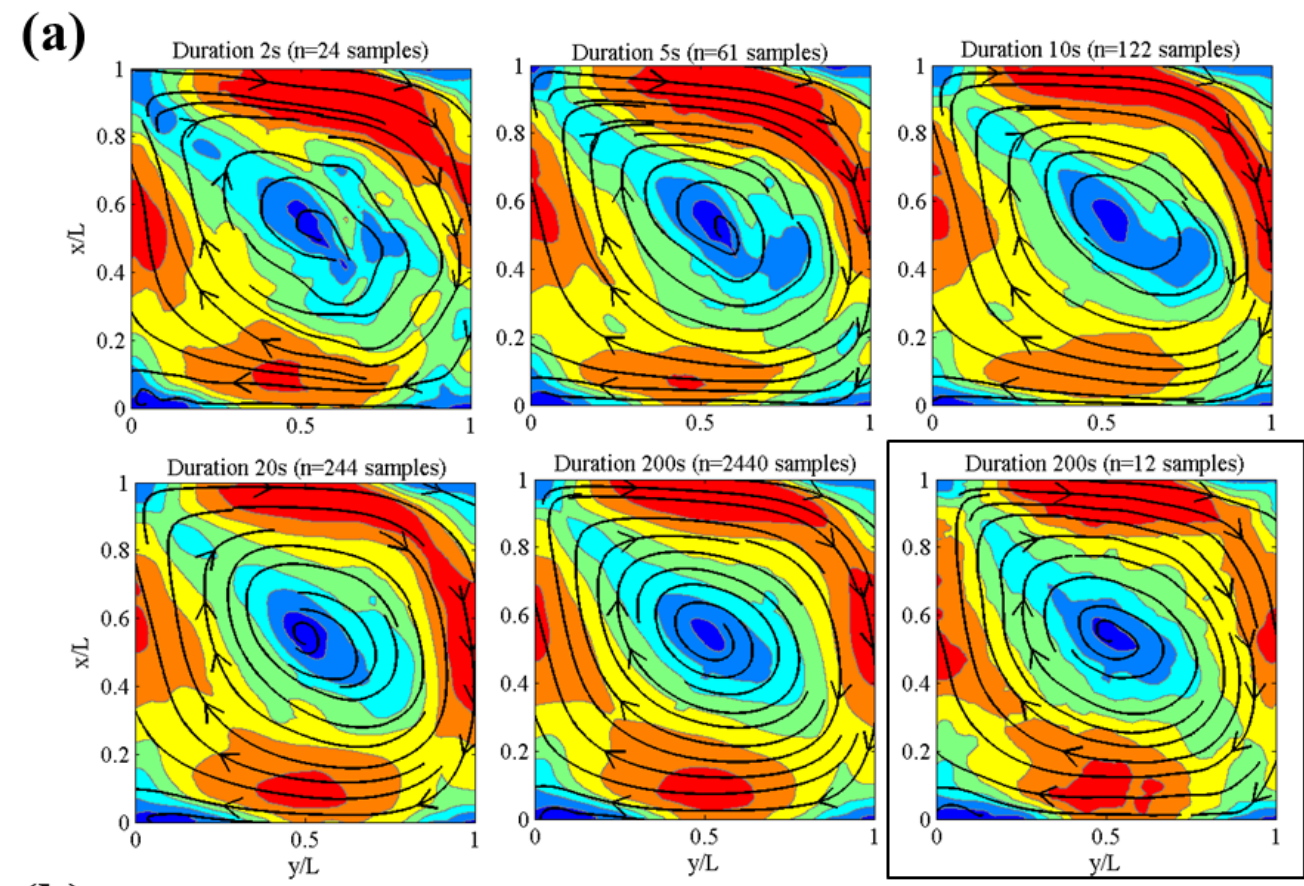

(b)

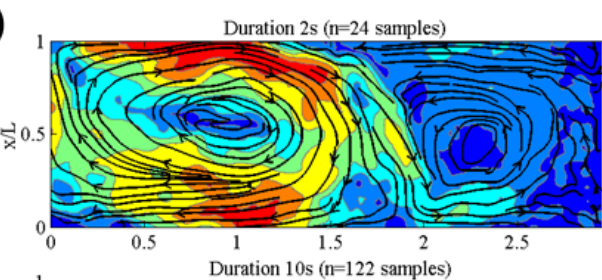

Duration $5 \mathrm{~s}$ ( $\mathrm{n}=61$ samples)
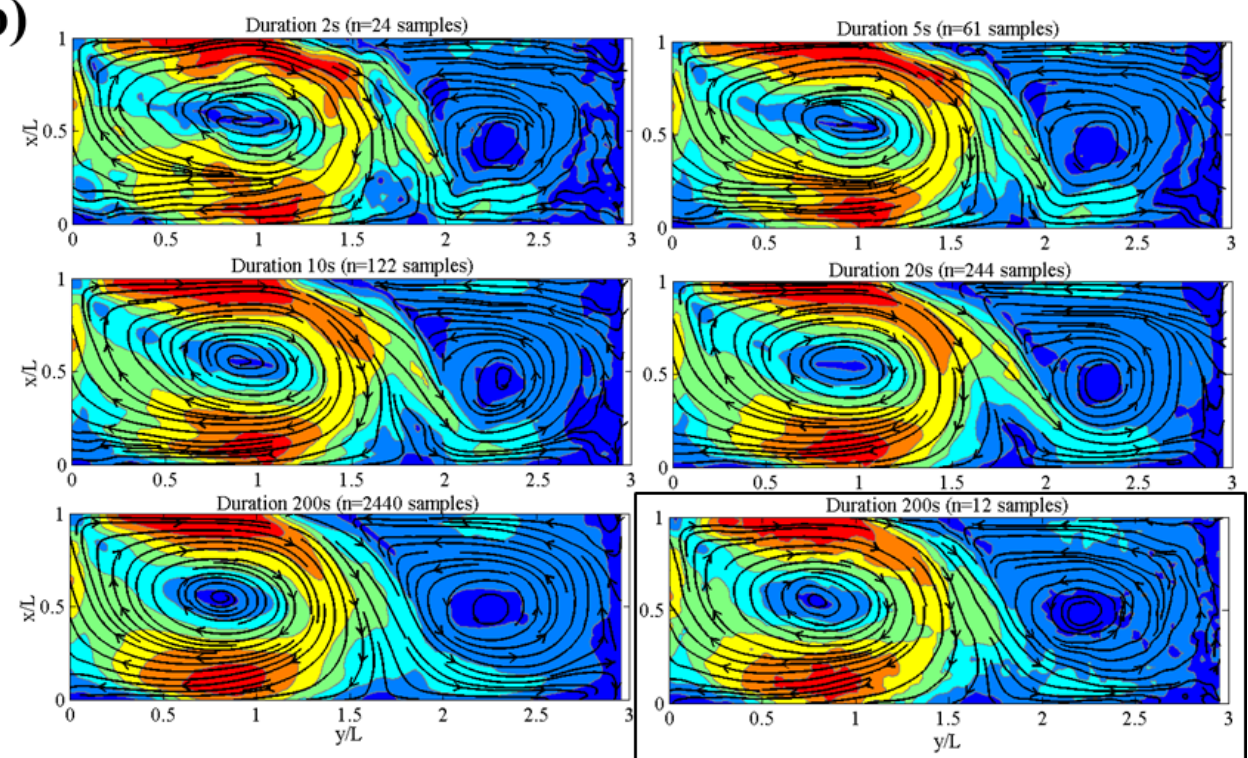

Velocity magnitude $(\mathrm{cm} / \mathrm{s})$

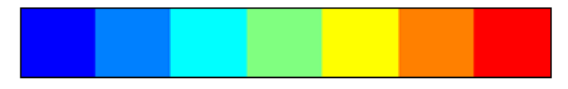

$\begin{array}{llllll}0.25 & 0.75 & 1.25 & 1.75 & 2.25 & 2.75\end{array}$

Figure 12. $2 D$ horizontal velocity fields measured at the elevation $z / h=0.71$ for configurations $W / L=1$ (a) and $W / L=3(b)$. For both configurations, the five first velocity fields are timely-averaged over increasing durations using the measurement sampling frequency and the last velocity field is averaged over 200s but with only 12 samples. 

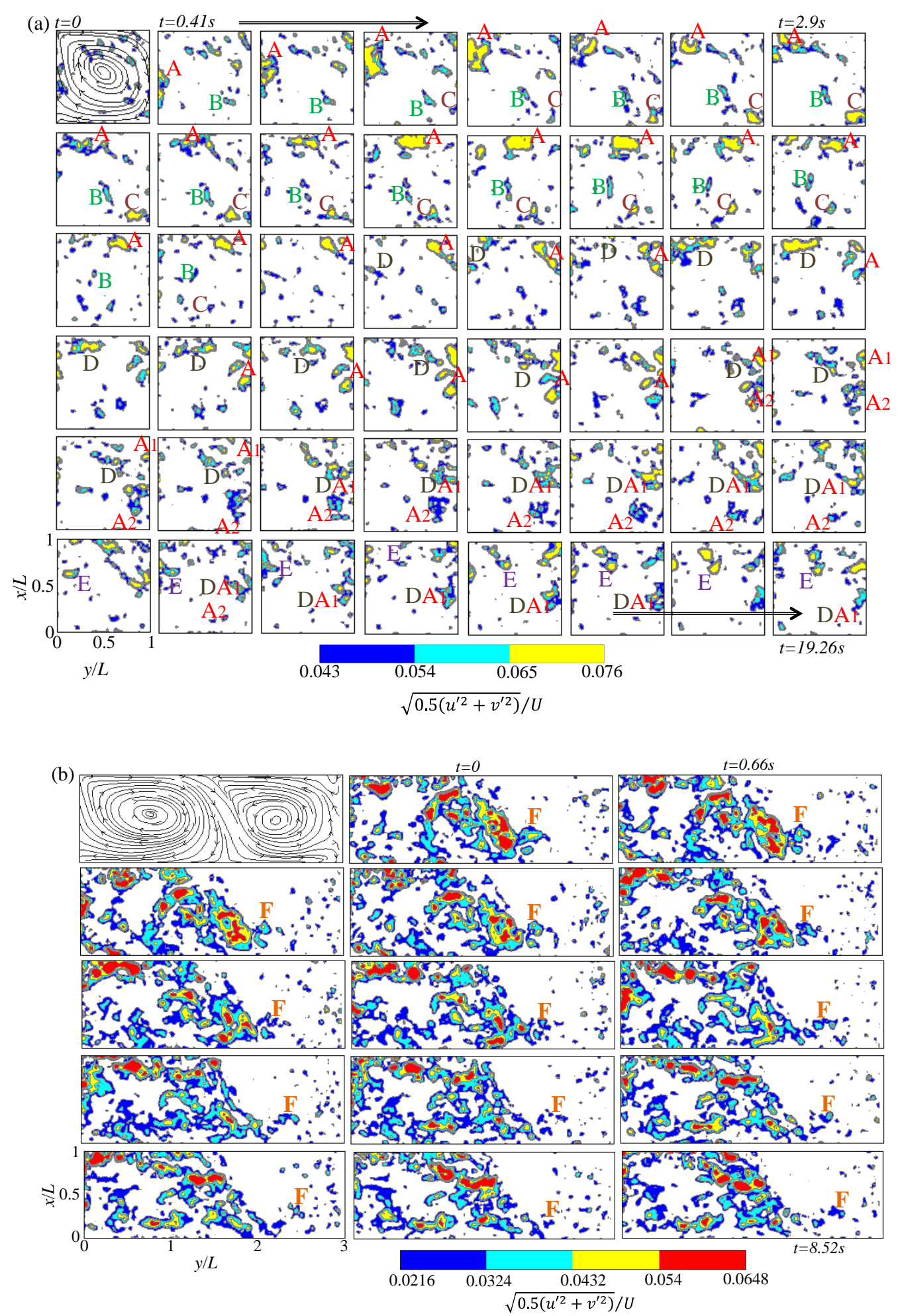

Figure 13. Spatial distribution of fluctuation velocity magnitude (averaged over 2 consecutive samples, that is $6.1 \mathrm{~Hz}$ ), plotted every 0.4 s for the configuration $W / L=1$ (a) and plotted every 0.66 s for the configuration $W / L=3(b)$. The prime sign refers to the fluctuating velocity component obtained through the Reynolds decomposition. Time increases from left to right and then returning to the following line. The streamlines corresponding to the fully converged mean flow are added on the top/left graphs. The letters refer to spots of high fluctuating velocity magnitudes. The colorbar differs for both graphs in order to detect the less-intense coherent structures in the second cell for $W / L=3$. 
(a)

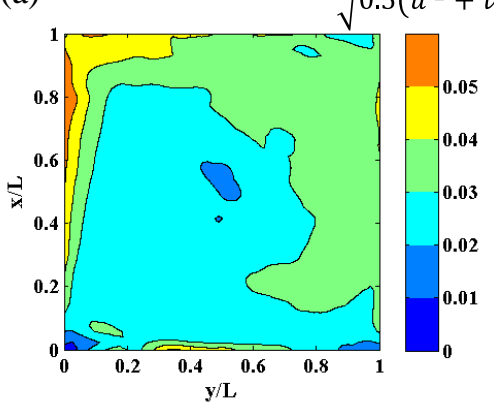

(b)

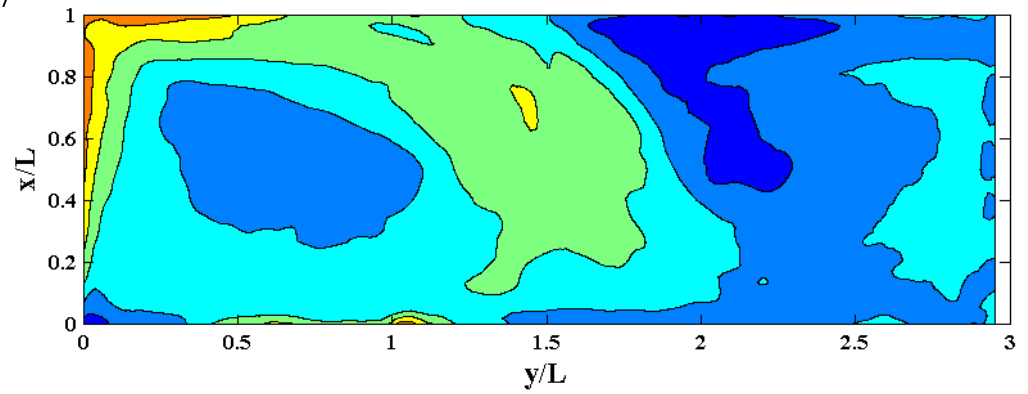

Figure 14. Spatial distribution of turbulent intensity within the cavity for the configuration $W / L=1$ (a) and $W / L=3(b)$ 